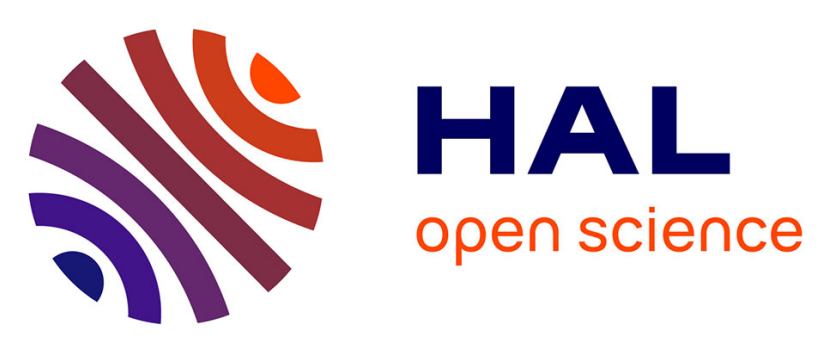

\title{
A new three-dimensional mixed finite element for direct numerical simulation of compressible viscoelastic flows with moving free surfaces
}

\author{
Luisa Silva, Rudy Valette, Patrice Laure, Thierry Coupez
}

\section{To cite this version:}

Luisa Silva, Rudy Valette, Patrice Laure, Thierry Coupez. A new three-dimensional mixed finite element for direct numerical simulation of compressible viscoelastic flows with moving free surfaces. International Journal of Material Forming, 2012, 5 (1), pp.55-72. 10.1007/s12289-011-1030-2 . hal00579571

HAL Id: hal-00579571

https://hal-mines-paristech.archives-ouvertes.fr/hal-00579571

Submitted on 26 Jul 2011

HAL is a multi-disciplinary open access archive for the deposit and dissemination of scientific research documents, whether they are published or not. The documents may come from teaching and research institutions in France or abroad, or from public or private research centers.
L'archive ouverte pluridisciplinaire HAL, est destinée au dépôt et à la diffusion de documents scientifiques de niveau recherche, publiés ou non, émanant des établissements d'enseignement et de recherche français ou étrangers, des laboratoires publics ou privés. 


\title{
A New Three-Dimensional Mixed Finite Element for Direct Numerical Simulation of Compressible Viscoelastic Flows with Moving Free Surfaces
}

\author{
Luisa Silva - Rudy Valette • Patrice Laure • \\ Thierry Coupez.
}

Received: date / Accepted: date

\begin{abstract}
A Mixed Finite Element (MFE) method for 3D non-steady flow of a viscoelastic compressible fluid is presented. It was used to compute polymer injection flows in a complex mold cavity, which involves moving free surfaces. The flow equations were derived from the Navier-Stokes incompressible equations, and we extended a mixed finite element method for incompressible viscous flow to account for compressibility (using the Tait model) and viscoelasticity (using a Pom-Pom like model).

The flow solver uses tetrahedral elements and a mixed velocity / pressure / extra-stress / density formulation, where elastic terms are solved by decoupling our system and density variation is implicitly considered. A new DEVSS-like method is also introduced naturally from the MINI-element formulation. This method has the great advantage of a low memory requirement. At each time slab, once the velocity has been calculated, all evolution equations (free surface and material evolution) are solved by a space-time finite element method. This method is a generalization of the discontinuous Galerkin method, that shows a strong robustness with respect to both re-entrant corners and flow front singularities.

Validation tests of the viscoelastic and free surface models implementation are shown, using literature benchmark examples. Results obtained in industrial 3D geometries underline the robustness and the efficiency of the proposed methods.
\end{abstract}

L. Silva, R. Valette, P. Laure, T. Coupez

Mines ParisTech, CEMEF - Centre de Mise en Forme des Matriaux

CNRS UMR 7635

BP 207, 1 rue Claude Daunesse

06904 Sophia Antipolis Cedex, France

Tel.: +33-493678903

Fax: +33492389752

E-mail: rudy.valette@mines-paristech.fr

P. Laure

Université de Nice Sophia Antipolis, Laboratoire J. A. Dieudonné

CNRS UMR 6621

Parc Valrose

06108 Nice Cedex 2, France 
Keywords Viscoelasticity · Compressibility - 3D Mixed Finite Element Methods · Free Surface Flows

\section{Introduction}

The need of accurate predictions of the material behavior in most physical problems, leads today to an increase on the complexity of the models considered, often reflected in the number of material parameters required to characterize a material. The higher is this degree of complexity, the wider will be the application field. This work focuses on 3D moving free surface flow computations of compressible viscoelastic fluids with enough efficiency to be applied on realistic industrial configurations. The underlying application of such development is the injection moulding simulation. This process involves three successive stages: filling, packing and cooling, that reveal different features of the behavior of the same material. Covering these successive steps without changing artificially the set of equations to be solved, embedding all the rheological response of the material all along the process, remains a challenging task. Towards this objective, the compressible viscoelasticity is a step forward as compressibility is definitely involved in the packing and cooling stage and viscoelasticity influences internal stresses, thermal behavior and crystallization $[1,2]$.

So far, a number of achievements has been done in terms of numerical methods that are stated here. Computational methods used in viscoelastic flows remain an active area of research [3]. Several methods can be used to solve viscoelastic macroscopic constitutive equations in complex flows [4-6] and three-dimensional applications can be found in $[7,8]$, but they did not take free surfaces into account. Two dimensional steady-state or transient viscoelastic flows with free surfaces have been studied for about 30 years, mainly applied to the die swell or filament stretching problems [9-15] and recently to extrudate swell or jet flow [16,17]. Most of the techniques are based on differential constitutive equations, since they are more efficient in CPU time and computational memory. In our work, we used a mixed finite element method and considered a time step marching scheme allowing to split the viscoelastic constitutive equation and the flow equations, the last ones being stabilized by a technique similar to the DEVSS method [18]. To have a molecular interpretation of the material behavior, the model chosen to establish the elastic contribution was the multi-mode Pom-Pom model [19]. An important feature of this model that justifies our choice is its correct description of the non-linear behavior both in elongation and in shear (even for linear and slightly branched polymers [20]) since 3D free surface flow shows features of both situations.

In the proposed approach, we also considered an Eulerian formulation, where the computational domain is divided in several subdomains, whose evolution is governed through a transport equation. Interfaces were accurately determined through an $r$-adaptation scheme [21]. We also considered that the same methods used to capture free surfaces on newtonian flows may be applied to more complex materials. Different approaches to the free surface determination problem have been proposed in the literature ([22] presents a review of the various existing methods and limitations), and the one considered in our work has been developed by one of the authors and co-workers [23].

We took into account the evolution of the material density with pressure and 
temperature. To account for compressibility, regarding what was done for viscous flows, various numerical methods have been proposed to solve compressible or incompressible problems, for what concerns independent variables, linear system solvers, and numerical stability. Several authors developed in the past unified computational methods for compressible and incompressible viscous flows [24-30], showing results for a wide range of flow speeds, but in two-dimensional simple geometries. Extensions of low to vanishing Mach number compressible flows to viscoelastic constitutive models have been studied by Webster and co-workers [32, 33,31 ] in a very comprehensive work, and were compared to experimental results in [34]. However, these studies were devoted to two-dimensional flows. Since our objective remains the possibility of application of this kind of models for threedimensional industrial applications, we have extended a previous approach involving weakly compressible flows of generalized newtonian fluids [35] to viscoelastic constitutive models. We split compressible and viscoelastic effects by considering that each brings its contribution to the stress determination: compressibility was taken into account classically through a state equation (the Tait law), whereas viscoelasticity was determined through the evolution of the extra-stress tensor.

The proposed formulation for the $3 \mathrm{D}$ non-steady flow of a viscoelastic compressible fluid will be presented as follows: in the first part, we outline the material behavior modelling, by drawing the basic features of the multi-mode Pom-Pom model, that has been implemented to treat the non-linear viscoelastic behavior, and how does the state law relates density evolution with pressure and temperature; in the next section, the computational methods used to solve the viscoelastic compressible flow problem are detailed. Two basic problems need to be treated: the presence of convective terms in constitutive equations, and strong non-linearity when we consider the coupled problem. A splitting method allowed separate resolution of the evolution equations (through a Space-Time Discontinuous Galerkin finite element method) from the flow equations, using mixed finite elements. Furthermore, extension to free surface, that is briefly described here, increased the complexity of our problem. Validation tests performed on 'benchmark' geometries (like the contraction or contraction/ expansion flow) are analyzed, and finally, three-dimensional free-surface flows are considered in simple and complex geometries.

\section{Physical and material behavior modelling}

Simulation of compressible viscoelastic flows with moving free surfaces requires the choice of physical and material behavior models. The purpose of the proposed model is to determine the evolution of velocity, pressure (and eventually temperature) fields over the whole computational domain, as well as the evolution of the region occupied by the fluid. The stress field distribution in the fluid will be explicitly determined from these variables. As one is dealing with a free surface flow, we considered that no stresses arise in the region not occupied by the fluid. For a question of simplification, let us consider the problem of one single polymer melt flowing with a free surface, neglecting mass and inertia forces. 
2.1 Volume occupied by the fluid

To identify the region occupied by the fluid, we used the same approach as presented in [23]. One uses a characteristic function $1_{\Omega_{f}}$ of the fluid region $\Omega_{f}$ evolving in the whole $3 \mathrm{D}$ domain $\Omega$ within the time interval $] 0, t_{e}\left[\right.$, where $t_{e}$ is the duration of the studied flow. In an Eulerian frame, a point $\vec{x}$ belongs to the fluid domain if the characteristic function at this point is equal to 1 . The characteristic function is consequently defined as:

$$
\forall t \in \mathbb{R}^{+} 1_{\Omega_{f}}(\vec{x}, t)=\left\{\begin{array}{l}
1 \text { if } \vec{x} \in \Omega_{f} \\
0 \text { if } \vec{x} \in \Omega \backslash \Omega_{f}
\end{array}\right.
$$

and it is then advected with the fluid velocity:

$$
\frac{\partial 1_{\Omega_{f}}}{\partial t}+\vec{v} \cdot \nabla 1_{\Omega_{f}}=0
$$

\subsection{Stress field distribution}

In the free surface problem, the stress field distribution approaches zero in $\Omega \backslash \Omega_{f}$ and continuity of velocity and normal stresses (when neglecting surface tension) at the interface must be satisfied.

As we have been considering a compressible viscoelastic flow, we need to specify the underlying assumptions of the physical model. Let us first remind that the purpose of this work is to develop a numerical solver for this type of flow and we did not intend to develop a proper thermodynamical model for compressible viscoelasticity. Indeed, the physical model should be considered as a simple but convenient way to account for both compressibility and viscoelasticity in polymer processing.

Consequently, we considered the Cauchy stress tensor in the fluid as an additive contribution :

$$
\sigma=-p I+\tau_{s}+\tau
$$

where $p$ is the pressure term, $\tau$ the viscoelastic extra-stress tensor and $\tau_{s}$ the viscous contribution in which the bulk viscosity is neglected. For a compressible flow, we used the classical equality for $\tau_{s}$ :

$$
\tau_{s}=2 \eta\left(\epsilon(\vec{v})-\frac{1}{3} \nabla \cdot \vec{v} I\right)
$$

where $\eta$ is the solvent viscosity (neglecting the bulk viscosity) and $\epsilon(\vec{v})-\frac{1}{3} \nabla \cdot \vec{v} I$ the deviatoric part of the strain rate tensor.

Conservation equations to be solved in $\Omega_{f}$ are:

$$
\left\{\begin{array}{l}
\nabla \cdot\left[2 \eta\left(\epsilon(\vec{v})-\frac{1}{3} \nabla \cdot \vec{v} I\right)\right]-\nabla p+\nabla \cdot \tau=0 \\
\nabla \cdot \vec{v}+\frac{1}{\rho} \frac{d \rho}{d t}=0 \\
+ \text { constitutive laws } \\
+ \text { boundary conditions }
\end{array} \text { in } \Omega_{f}\right.
$$


The strongest assumption on the physical model concerned the pressure term. When considering incompressible viscoelastic behavior, the pressure term can be seen as an arbitrary spherical contribution. When adding compressibility, considered here as the immediate resistance of the fluid to a change of volume, one has to use a state law linking pressure to density. Consequently, $p$ must be defined carefully. We considered here that, starting from rest, $\tau$ would remain equal to zero for any homogeneous volume variation. Implications are detailed in the two next paragraphs.

We also assume that, since polymers are weakly compressible, problem (5) can be seen has a quasi-static one. This implies that a splitting technique can be used for its numerical resolution, and it will be detailed in section 3 .

\subsection{Compressibility modelling: the state law}

In this paper, we restricted the study to isothermal flows. However, in injection moulding, compressible effects are crucial in both packing and cooling stages, as pressure allows to compensate for thermal shrinkage. It is then obvious that both isothermal compressibility and thermal expansion coefficient must appear in the state law. A general state equation (also known as PVT relationship) describes dependence between specific volume $v$ (or density $\rho$ ), pressure $p$, and temperature $T$. If one considers each variation independently, one can define: the isothermal compressibility, $\chi_{p}$, measuring the compressibility due to pressure variation, and the thermal expansion coefficient, $\chi_{T}$, which are positive quantities, proper to each material:

$$
\begin{aligned}
& \chi_{p}=-\frac{1}{v}\left(\frac{\partial v}{\partial p}\right)_{T}=\frac{1}{\rho}\left(\frac{\partial \rho}{\partial p}\right)_{T}, \\
& \chi_{T}=\frac{1}{v}\left(\frac{\partial v}{\partial T}\right)_{p}=-\frac{1}{\rho}\left(\frac{\partial \rho}{\partial T}\right)_{p} .
\end{aligned}
$$

By differentiating the state law, one obtains:

$$
-\frac{d v}{v}=\chi_{p} d p-\chi_{T} d T=\frac{d \rho}{\rho}
$$

that allows to represent the time evolution of the density for a given material as:

$$
\frac{1}{\rho} \frac{d \rho}{d t}=\chi_{p} \frac{d p}{d t}-\chi_{T} \frac{d T}{d t}
$$

To determine $\chi_{p}$ and $\chi_{T}$, we choosed the Tait law [36], which is classically used in polymer engineering:

$$
v(T, p)=v_{0}(T)\left[1-C \ln \left(1+\frac{p}{B(T)}\right)\right]+v_{t}(T, p)
$$

where $C=0.0894$ in a universal constant. This equation describes polymer compressible behavior both in the liquid as in the solid state, by representing differently $v_{0}(T)$ and $B(T)$ for each phase. However, as already mentioned, we restricted the present study to isothermal flows, using a given temperature $T . v_{t}(T, p)$ is an additional term to handle the sharp density change in the vicinity of $T_{t}$ for semicrystalline polymers. In the cavity's regions filled with air, we considered an isothermal behaviour, with a constant isothermal compressibility coefficient. 
2.4 Viscoelasticity modelling: the Pom-Pom constitutive equation

We have used a Pom-Pom like constitutive equation for the viscoelastic model. The choice of this model was motivated by the fact that one can clearly identify orientation and stretch effects on the stress field. As previously explained, we expected the viscoelastic model not to perturb the compressible behavior in a compression deformation. The Pom-Pom model, introduced by McLeish and Larson [19] to describe the behavior of branched polymers, has been well studied in recent years. The dXPP model, which is a modification of the original differential Pom-Pom model, has been proposed by [37]. We will limit ourselves to its short description. Particular features of the dXPP model, compared to the original differential Pom-Pom model are:

- orientation equation is no more of Maxwellian type, which reduces numerical problems when solving it for high elongation rates,

- it includes local branch point displacement, a modification of the stretch relaxation time to account for local motion of the branch point,

- it exhibits a second normal stress difference in shear.

When dropping this last property, the multimode dXPP model can be simplified to:

$$
\begin{gathered}
\tau=\sum_{i=1}^{m} G_{0 i}\left(3 \lambda_{i}^{2} s_{i}-I\right) \\
\frac{\partial s_{i}}{\partial t}+\vec{v} \cdot \nabla s_{i}-\left[\nabla \vec{v} s_{i}+s_{i} \nabla \vec{v}^{T}\right]+2\left[s_{i}: \epsilon(\vec{v})\right] s_{i}+\frac{1}{\theta_{b i}}\left(s_{i}-\frac{I}{d}\right)=0 \\
\frac{\partial \lambda_{i}}{\partial t}+\vec{v} \cdot \nabla \lambda_{i}-\lambda_{i}\left[\epsilon(\vec{v}): s_{i}\right]+\frac{1}{\theta_{s i}}\left(\lambda_{i}-1\right) e^{\nu_{i}\left(\lambda_{i}-1\right)}=0
\end{gathered}
$$

where $s_{i}$ and $\lambda_{i}$ are respectively the orientation tensor and the stretch variable of mode $i$, and $m$ is the number of modes. There are four parameters for each mode: $G_{0}$ is an elastic modulus, and $\theta_{b}, \theta_{s}$ and $\nu$ can be seen, respectively, as a reptation time, a retraction time and a measure of the influence of the surrounding polymer chains on the backbone tube stretch (it is generally taken as $\nu=2 / q$ where $q$ is the number of arms of the branched polymer).

Using this model, for small values of compressibility, one can check that no viscoelastic stresses arise from a pure compression deformation, as the orientation tensor remains unchanged and the stretch dynamic activation is negligible. However, parameters are dependent on temperature $T$.

\section{Computational methods}

3.1 A 4-field mixed finite element method for the viscoelastic compressible flow problem

For sake of simplicity, let us consider in this section the model described in the previous section, applied to one single domain, compressible and one-mode viscoelastic fluid for which we neglect the stretch dynamics (so that $\lambda=1$ ). The weak formulation of the proposed problem is found by considering its product by test functions 
chosen in adequate spaces, and is thus written as: find $(\vec{v}, p, s, \rho) \in \mathcal{V} \times \mathcal{P} \times \mathcal{S} \times \mathcal{L}$ such that

$$
\left\{\begin{array}{l}
\int_{\Omega} 2 \eta\left[\epsilon(\vec{v})-\frac{1}{3} \operatorname{tr}(\epsilon(\vec{v})) I\right]: \epsilon(\vec{w}) d \Omega \\
\quad-\int_{\Omega} p \nabla \cdot \vec{w} d \Omega+\int_{\Omega}\left[G_{0}(3 s-I)\right]: \epsilon(\vec{w}) d \Omega=0 \\
\int_{\Omega} q \nabla \cdot \vec{v} d \Omega+\int_{\Omega} q \frac{1}{\rho} \frac{d \rho}{d t} d \Omega=0 \\
\int_{\Omega} \phi:\left[\frac{\partial s}{\partial t}+\mathbf{v} \cdot \nabla s-\left[\nabla \mathbf{v} s+s \nabla \mathbf{v}^{T}\right]+2[s: \epsilon(\vec{v})] s+\frac{1}{\theta_{b i}}\left(s-\frac{I}{d}\right)\right] d \Omega=0 \\
\int_{\Omega} \psi\left(\frac{1}{\rho} \frac{d \rho}{d t}-\chi_{p} \frac{d p}{d t}\right) d \Omega=-\int_{\Omega} \psi \chi_{T} \frac{d T}{d t} d \Omega
\end{array}\right.
$$

$\forall(\vec{w}, q, \phi, \psi) \in \mathcal{V} \times \mathcal{P} \times \mathcal{S} \times \mathcal{L}$

$\mathcal{V}, \mathcal{P}, \mathcal{S}$ and $\mathcal{L}$ are the functional spaces:

$$
\begin{aligned}
& \mathcal{V}=\left\{\vec{v} \in H^{1}(\Omega)^{3} \times\right] 0, t_{e}\left[, \int_{0}^{t_{e}}\|\vec{v}\|_{H^{1}(\Omega)^{3}}^{2} d t<\infty\right\} \\
& \mathcal{P}=\left\{p \in H^{1}(\Omega) \times\right] 0, t_{e}[\} \\
& \mathcal{S}=\left\{s \in L^{2}(\Omega)^{3 \times 3} \times\right] 0, t_{e}\left[, s_{i j}=s_{j i}\right\} \\
& \mathcal{L}=\left\{\rho \in L^{2}(\Omega) \times\right] 0, t_{e}[\}
\end{aligned}
$$

Let us remark that the pressure space $\mathcal{P}$ has to be richer than in the standard incompressible Stokes problem, as a term $\nabla p$ is involved in the compressible formulation [35].

Let $\Omega_{h}$ be a discretization of our domain in space-time finite-elements. As in a previous work [23], we considered a structured mesh on time: each element is the cartesian product between the simplex $K$ and a time slab $] t_{i}, t_{i+1}[$. Furthermore, let us consider that $\vec{v}, p$ and $s$ are discontinuously interpolated in time, with a polynomial of degree $n$. Considering spatial discretization, $(\vec{v}, p, s)$ must satisfy stability conditions: finite element approximation of the velocity and extra-stress must verify the inf-sup condition [38]. In the proposed approximation, we chose a linear interpolation for velocity and pressure with bubble enrichment for velocity, the $P 1+/ P 1$ element, also referred as the MINI-element [40], even if only a $\mathrm{P} 1 / \mathrm{P} 1$ interpolation is necessary in the compressible case [41]. A discontinuous $P 0$ interpolation for extra-stresses was chosen, as well as for density. Fortin and coworkers [42] suggest the same subspace approximations for pressure and density, but a lower order interpolation for density will allow us condensation, and therefore a decrease on the number of degrees of freedom. This new space-time discontinuous Galerkin method is an extension of classical discontinuous Galerkin method, where stabilization and inf-sup conditions are respectively satisfied by upwinding, jump terms and choice of approximation spaces. This method is an alternative to recently (in the case of viscoelastic flow computations) proposed stabilization methods such as continuous four-field Galerkin/least-squares [39]. Approximations subspaces are defined as:

$$
\begin{aligned}
& \mathcal{V}_{h}=\left\{\vec{v}_{h} \in H^{1}(\Omega)^{3}, \vec{v}_{h} \in \mathrm{P} 1(K)^{3}, \vec{v}_{h} \in \mathrm{P} 0(] t_{i}, t_{i+1}[)\right\} \\
& \mathcal{B}_{h}=\left\{\vec{b}_{h} \in H^{1}(\Omega)^{3}, \vec{b}_{h} \in \mathrm{P} 1\left(K_{F}\right)^{3}, \vec{b}_{h}=\overrightarrow{0}_{0} \text { on } \partial K, \vec{b}_{h} \in \mathrm{P} 0(] t_{i}, t_{i+1}[)\right\} \\
& \mathcal{P}_{h}=\left\{p_{h} \in H^{1}(\Omega), p_{h} \in \mathrm{P} 1(K), p_{h} \in \mathrm{P} 0(] t_{i}, t_{i+1}[)\right\} \\
& \mathcal{S}_{h}=\left\{s_{h} \in L^{2}(\Omega)^{3 \times 3}, s_{h} \in \operatorname{P} 0(K)^{3 \times 3}, s_{h} \in \operatorname{Pn}(] t_{i}, t_{i+1}[)\right\} \\
& \mathcal{L}_{h}=\left\{\rho_{h} \in L^{2}(\Omega), \rho_{h} \in \operatorname{P} 0(K), \rho_{h} \in \operatorname{Pn}(] t_{i}, t_{i+1}[)\right\}
\end{aligned}
$$


where $K_{F}$ is the sub-element formed by joining face $F$ to the center of $K$ (pyramidal bubble function) and $P 1(K)$ represents an interpolation function of degree equal or inferior to 1 on $K$. The discrete variational formulation is written: find $\left(\vec{v}_{h}+\right.$ $\left.\vec{b}_{h}, p_{h}, s_{h}, \rho_{h}\right) \in\left(\mathcal{V}_{h} \oplus \mathcal{B}_{h}\right) \times \mathcal{P}_{h} \times \mathcal{S}_{h} \times \mathcal{L}_{h}$ such that

$$
\left\{\begin{array}{l}
\int_{\Omega_{h}} 2 \eta\left[\epsilon\left(\vec{v}_{h}\right)-\frac{1}{3} \operatorname{tr}\left(\epsilon\left(\vec{v}_{h}\right)\right) I\right]: \epsilon\left(\vec{w}_{h}\right) d \Omega \\
\quad-\int_{\Omega_{h}} p_{h} \nabla \cdot \vec{w}_{h} d \Omega+\int_{\Omega_{h}} G_{0}\left(3 s_{h}-I\right): \epsilon\left(\vec{w}_{h}\right) d \Omega=0 \\
\int_{\Omega_{h}} 2 \eta\left[\epsilon\left(\vec{b}_{h}\right)-\frac{1}{3} \operatorname{tr}\left(\epsilon\left(\vec{b}_{h}\right)\right) I\right]: \epsilon\left(\vec{b}_{h}^{*}\right) d \Omega \\
\quad-\int_{\Omega_{h}} p_{h} \nabla \cdot \vec{b}_{h}^{*} d \Omega+\int_{\Omega_{h}} G_{0}\left(3 s_{h}-I\right): \epsilon\left(\vec{b}_{h}^{*}\right) d \Omega=0 \\
\int_{\Omega_{h}} q_{h} \nabla \cdot \vec{v}_{h} d \Omega+\int_{\Omega_{h}} q_{h} \nabla \cdot \vec{b}_{h} d \Omega+\int_{\Omega_{h}} q_{h} \frac{1}{\rho_{h}} \frac{d \rho_{h}}{d t} d \Omega=0 \\
\int_{\Omega_{h}} \phi_{h}:\left[\frac{\partial s_{h}}{\partial t}+\vec{v}_{h} \cdot \nabla s_{h}-\left[\nabla \vec{v}_{h} s_{h}+s_{h} \nabla \vec{v}_{h}^{T}\right]\right. \\
\left.\quad+2\left[s_{h}: \epsilon\left(\vec{v}_{h}\right)\right] s_{h}+\frac{1}{\theta_{b i}}\left(s_{h}-\frac{I}{d}\right)\right] d \Omega=0 \\
\int_{\Omega_{h}} \psi_{h}\left(\frac{1}{\rho_{h}} \frac{d \rho_{h}}{d t}-\chi_{p} \frac{d p_{h}}{d t}\right) d \Omega=-\int_{\Omega_{h}} \psi_{h} \chi_{T} \frac{d T_{h}}{d t} d \Omega
\end{array}\right.
$$

$\forall\left(\vec{w}_{h}+\vec{b}_{h}^{*}, q_{h}, \phi_{h}, \psi_{h}\right) \in\left(\mathcal{V}_{h} \oplus \mathcal{B}_{h}\right) \times \mathcal{P}_{h} \times \mathcal{S}_{h} \times \mathcal{L}_{h}$. Using a matrix notation, we are led to the following type of subsystem in each element $K$, being $V, V_{b}, P, s, \rho$ the unknowns in the element:

$$
\left(\begin{array}{ccccc}
A_{v v} & 0 & B_{v p} & B_{v s} & 0 \\
0 & A_{b b} & B_{b p} & B_{b s} & 0 \\
B_{v p}^{t} & B_{b p}^{t} & 0 & 0 & B_{p \rho} \\
B_{s v} & 0 & 0 & A_{s s} & 0 \\
0 & 0 & B_{\rho p} & 0 & A_{\rho \rho}
\end{array}\right)\left(\begin{array}{c}
V \\
V_{b} \\
P \\
s \\
\rho
\end{array}\right)=\left(\begin{array}{c}
F_{v} \\
0 \\
0 \\
F_{s} \\
F_{\rho}
\end{array}\right)
$$

Matrices $A_{v v}, A_{b b}, B_{v p}, B_{b p}$ are similar to the matrices obtained for the Stokes incompressible problem. To get as closer as possible to a resolution similar to Stokes [43] we need to condensate the bubble function, the variation of density and the extra-stress.

To condensate the bubble, we proceeded classically:

$$
\begin{aligned}
& A_{b b} V_{b}+B_{b b} P+B_{b s} s=0 \\
& \quad \Longrightarrow V_{b}=-A_{b b}^{-1}\left(B_{b p} P+B_{b s} s\right)
\end{aligned}
$$

Condensation of the density evolution was also straightforward and done through the last equation:

$$
A_{\rho \rho} \rho+B_{\rho p} P=F_{\rho} \Longrightarrow \rho=A_{\rho \rho}^{-1}\left(F_{\rho}-B_{\rho p} P\right)
$$

On the other hand, condensation of the extra-stress seems more difficult since it depends on orientation, that follow non-linear equations with terms that depend on velocity and on orientation itself. Let us start by focusing on the computation of the submatrix $B_{v s}$. Since $s$ has been chosen constant on each element, and velocity is linear, we can write:

$$
\int_{\Omega_{h}} s_{h}: \epsilon\left(\vec{w}_{h}\right) d \Omega=\sum_{K \subset \Omega_{h}} \int_{K} s_{K}: \epsilon\left(\vec{w}_{K}\right) d \Omega
$$


Applying Green's formula:

$$
\begin{aligned}
& \int_{\Omega_{h}} s_{h}: \epsilon\left(\vec{w}_{h}\right) d \Omega \\
& =\sum_{K \subset \Omega_{h}} \int_{\partial K}\left(s_{K} \cdot \vec{n}\right) \vec{w} d \Gamma \\
& =\sum_{K \subset \Omega_{h}} \sum_{F \in \partial K}[s]_{K}^{F} \cdot \vec{n}_{K}^{F} \int_{F} \vec{w} d \Gamma
\end{aligned}
$$

In these equations, $K$ means the value on element $K,[s]_{K}^{F}$ is the jump of $s_{K}$ at the face $F$ and is defined as

$$
[s]_{K}^{F}=s_{K}-s_{K(F)}
$$

where $s_{K(F)}$ is the value of $s$ on the adjacent element to the face $F$ of $K, \partial K$ is the boundary of the element $K, \vec{n}$ is the outward unit normal vector to $\partial K$.

Using the properties of subspace $\mathcal{B}_{h}$, one concludes that

$$
\int_{\Omega_{h}} s_{h}: \epsilon\left(\vec{b}_{h}^{*}\right) d \Omega=0
$$

since

$$
\int_{\partial K} \vec{b}_{h} d \Gamma=0
$$

One the concludes that submatrices $B_{b s}$ may be eliminated and equation (19) is reduced to:

$$
V_{b}=-A_{b b}^{-1} B_{b p} P
$$

Extra-stress is then easier to condensate:

$$
B_{s v} V+A_{s s} s=F_{s} \Longrightarrow s=A_{s s}^{-1}\left(F_{s}-B_{s v} V\right)
$$

We were finally led to the much simpler 2-field formulation:

$$
\left(\begin{array}{cc}
\hat{A} & B_{v p} \\
B_{v p}^{t} & \tilde{A}
\end{array}\right)\left(\begin{array}{c}
V \\
P
\end{array}\right)=\left(\begin{array}{c}
F_{v}-B_{v s} A_{s s}^{-1} F_{s} \\
-B_{p \rho} A_{\rho \rho}^{-1} F_{\rho}
\end{array}\right)
$$

where $\hat{A}=A_{v v}-B_{v s} A_{s s}^{-1} B_{s v}$ and $\tilde{A}=-B_{b p}^{t} A_{b b}^{-1} B_{b p}-B_{p \rho} A_{\rho \rho}^{-1} B_{\rho p}$.

Even though our 2-field problem seems simple to solve, a few points need to be outlined:

- density can be completely eliminated from our system. $A_{\rho \rho}$ is simply a mass matrix, but $B_{p \rho} A_{\rho \rho}^{-1} B_{\rho p}$ remains non-symmetric and depending on velocity and pressure, giving therefore a non-linear character to our system. If we take velocity at the previous time step, over the element $K$, this submatrix will be:

$$
\begin{gathered}
B_{p \rho} A_{\rho \rho}^{-1} B_{\rho p}=\frac{1}{\Delta_{t}} \int_{K} \chi_{p} N_{K}^{p} N_{K}^{p} d \Omega \\
-\sum_{F \in \partial K} \int_{F}(\mathbf{v} \cdot \vec{n})^{-} d \Gamma \int_{K} \chi_{p}\left(N_{K}^{p}-N_{K(F)}^{p}\right) d \Omega
\end{gathered}
$$


$\vec{n}$ is the outward unit normal to face $F$ and $(\mathbf{v} \cdot \vec{n})^{-}$is the outward flux of this same face. $N_{K}^{p}$ is the test function for the pressure $p$ at element $K$ and $N_{K(F)}^{p}$ on the neighbor element $K(F)$.

- in what concerns extra-stress, even though matrix $A_{s s}$ is diagonal, several difficulties arise from this formulation: non-symmetry of $B\left(B_{s v} \neq B_{v s}^{t}\right)$; convective terms for $B$ and evolution equations (which are easier to treat independently), strong non-linearity of $s$ which a function of $\vec{v}$ and $s$, and in the general case, one has to treat also the stretch equation.

At this point we had to choose between solving a coupled or decoupled system. In the first case, the extra-stress tensor would be computed by splitting the system in two sub-systems: knowing the configuration of our computational domain (which means knowing its molecular orientation distribution and stretch) one solves conservation equations then, knowing $\vec{v}$ and $\mathrm{p}$, one calculates extra-stress $\tau$. Moreover, coupling the equations allows the simultaneous resolution of the discretized governing equations for the whole set of primary variables by means of an iterative scheme of the Newton type. However, very large problems and generalization to 3D and multimode computations seem difficult.

Alternatively, an advantage of decoupling is that the global problem, with a large number of unknowns, and therefore large-consuming memory, is broken up into smaller systems: a Stokes compressible problem perturbed by an elastic term (treated as a known body force term) and the non-linear evolution equations that allow the determination of $\tau$. Furthermore, each problem can be treated taking into account its mathematical type: the Stokes problem is elliptic and linear, whereas orientation and stretch problems involve non-linearities and convection. Disadvantages are only related to the need of a marching time scheme of both calculations, which could lead to time instabilities if not carefully carried out. Therefore, we used a decoupled method.

Finally, to synthesize the proposed solution scheme, on one time slab $I=$ $t_{i}, t_{i+1}\left[\right.$, the Stokes problem was solved determining $\left(\vec{V}_{I}, P_{I}\right)$ constant on this interval for a known extra-stress configuration $\left(s_{I}, \lambda_{I}\right)$. Afterwards, orientation and stretch were updated $\left(s_{I}, \lambda_{I}\right)$ with $\vec{V}_{I}$.

\subsection{Using bubble function as stabilisation}

In the proposed formulation, a solvent viscosity was included, arbitrary, that may result in a solution too close of a Newtonian behavior. Some authors usually completely eliminate it, by using stabilization methods like the EVSS or the DEVSS formulations. In the present case, solvent viscosity can be chosen very small because our bubble stabilization procedure is approaching the DEVSS method of Guénette and Fortin [18]. Applying this method to the simple incompressible viscoelastic flow problem led to introduce an additional unknown $H$ as a representation of the strain rate tensor and find $(\vec{v}, p, \tau, H)$ such that:

$$
\left\{\begin{array}{l}
\nabla \cdot[2 \eta \epsilon(\vec{v})]-\nabla p+\nabla \cdot \tau \\
\quad+\left(\nabla \cdot\left[2 \eta_{s t a b} \epsilon(\vec{v})\right]-\nabla \cdot\left[2 \eta_{s t a b} H\right]\right)=0 \\
\nabla \cdot \vec{v}=0 \\
H-\epsilon(\vec{v})=0
\end{array}\right.
$$


where $\eta$ is the solvent viscosity and $\eta_{\text {stab }}$ is used as a stabilizing parameter and $H$ will be a different representation of the strain rate tensor in the discrete version of equation (30), as mentioned by Guénette and Fortin [18]. Let us consider $\vec{u}_{h}=$ $\vec{v}_{h}+\vec{b}_{h}$. The discrete variational form for this problem is: find $\left(\vec{u}_{h}, p_{h}, \tau_{h}, H_{h}\right) \in$ $\left(\mathcal{V}_{h} \oplus \mathcal{B}_{h}\right) \times \mathcal{P}_{h} \times \mathcal{S}_{h} \times \mathcal{H}_{h}$ such that

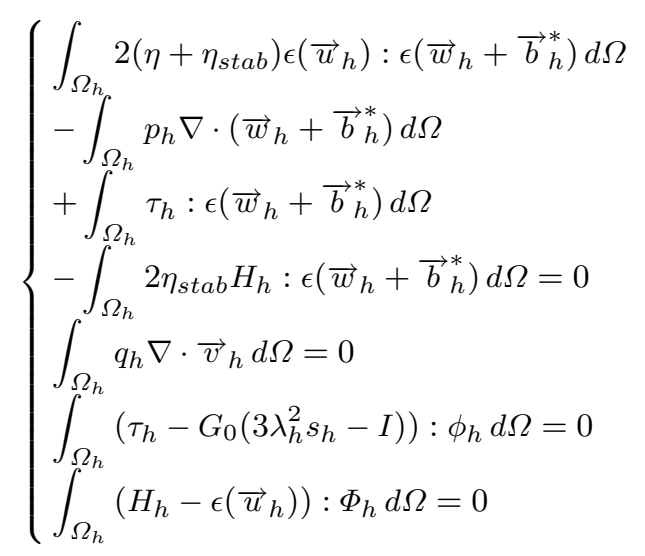

$\forall\left(\vec{w}_{h}+\vec{b}_{h}^{*}, q_{h}, \phi_{h}, \Phi_{h}\right) \in\left(\mathcal{V}_{h} \oplus \mathcal{B}_{h}\right) \times \mathcal{P}_{h} \times \mathcal{S}_{h} \times \mathcal{H}_{h}$. The last equation allowed us to consider $H_{h}$ as the projection of $\epsilon\left(\vec{u}_{h}\right)$ in $\mathcal{H}_{h}$ :

$$
H_{h}=\mathcal{P}_{\mathcal{H}_{h}} \epsilon\left(\vec{u}_{h}\right)
$$

where $\mathcal{P}_{\mathcal{H}_{h}}$ is the projection operator. As the subspace $\mathcal{H}_{h}$ must not be entirely representative of the space defining $\epsilon\left(\vec{v}_{h}\right)$ [18], we then simply considered the linear part of the velocity interpolation, $\vec{v}_{h}\left(\mathcal{H}_{h}=\mathcal{V}_{h}\right)$ :

$$
H_{h}=\epsilon\left(\vec{v}_{h}\right)
$$

The first equation in (31) may then be re-written as:

$$
\begin{aligned}
& \int_{\Omega_{h}} 2 \eta \epsilon\left(\vec{v}_{h}\right): \epsilon\left(\vec{w}_{h}\right) d \Omega \\
& +\int_{\Omega_{h}} 2\left(\eta+\eta_{s t a b}\right) \epsilon\left(\vec{b}_{h}\right): \epsilon\left(\vec{b}_{h}^{*}\right) d \Omega \\
& -\int_{\Omega_{h}} p \nabla \cdot \vec{w}_{h} d \Omega-\int_{\Omega_{h}} p \nabla \cdot \vec{b}_{h}^{*} d \Omega \\
& +\int_{\Omega_{h}} \tau_{h}: \epsilon\left(\vec{w}_{h}\right) d \Omega+\int_{\Omega_{h}} \tau_{h}: \epsilon\left(\vec{b}_{h}^{*}\right) d \Omega=0
\end{aligned}
$$

which is similar to the initially proposed formulation, where we added a stabilizing viscosity, without affecting the final result.

3.3 The STDG method applied to the evolution equations

Once the velocity field was established, we need to determine orientation and stretch (as well as free surface evolution). These evolution equations were solved through a Space-Time Discontinuous Galerkin method. Basically, Space-Time means 
that it allows a simultaneous resolution in space and in time $(\vec{x}=(x, t))$ and the computational domain is $\left.\left(\Omega^{x} \times\right] 0, t_{e}[)\right)$, where $t_{e}$ is the duration of the process studied); Discontinuous Galerkin means that we choose discontinuous interpolations both in space (low order) and in time (higher order).

In this case, we considered a $(d+1)$ finite element mesh, where $d$ is the spatial dimension, unstructured in space and structured in time. On the element $K$, orientation and stretch were interpolated by functions constant and discontinuous in space, $P 0(K)$, and polynomial (degree $n)$ and discontinuous in time $\left(P n(] t_{i}, t_{i+1}[)\right)$. The case $n=0$ corresponds to the classical Discontinuous Galerkin method. Subspaces of approximation for orientation and stretch are $\mathcal{S}_{h}$ and $\mathcal{L}_{h}$, defined previously. Hence, orientation and stretch may be interpolated through the whole element $K$ as

$$
\begin{aligned}
& s_{K}(t)=\sum_{p=0}^{n} s_{K}^{p}\left(t-t_{i}\right)^{p} \\
& \lambda_{K}(t)=\sum_{p=0}^{n} \lambda_{K}^{p}\left(t-t_{i}\right)^{p}
\end{aligned}
$$

where $s_{K}^{p}$ is the interpolation of $s$ over $K$, unknown of our problem. The discrete variational form of the problems is: find $s_{h} \in \mathcal{S}_{h}$ and $\lambda_{h} \in \mathcal{L}_{h}$ such that

$$
\begin{aligned}
& \int_{\Omega}\left(\frac{\partial s_{h}}{\partial t}+\vec{v} \cdot \nabla s_{h}-\left[\nabla \vec{v} s_{h}+s_{h} \nabla \vec{v}^{T}\right]+2\left[s_{h}: \epsilon(\vec{v})\right] s_{h}+\frac{1}{\theta_{b}} s_{h}\right) \Phi_{h} d \Omega \\
& =\int_{\Omega} \frac{I}{3 \theta_{b}} \Phi_{h} d \Omega \\
& \int_{\Omega}\left(\frac{\partial \lambda_{h}}{\partial t}+\vec{v} \cdot \nabla \lambda_{h}-\lambda_{h}\left[\epsilon(\vec{v}): s_{h}\right]+\frac{e^{\nu\left(\lambda_{h}-1\right)}}{\theta_{s}}\left(\lambda_{h}-1\right)\right) \varphi_{h} d \Omega=0
\end{aligned}
$$

$\forall \Phi \in \mathcal{S}_{h}$ and $\forall \varphi \in \mathcal{L}_{h}$. Before introducing the final system to solve, let us remark that since $s$ is symmetrical the total number of unknowns is:

$$
\sum_{K \subset \Omega_{h}} \frac{d(d+1)}{2}(n+1)+(n+1)
$$

where $n$ is the interpolation order in time. To solve our problem, we split the resolution into two simpler problems: first we determined the stretch level, afterwards we update orientation. We assembled the contribution of each element to a global matrix.

\subsection{Moving free surfaces}

In order to calculate a moving free surface (typically a material front in a 3D mould in injection process), we have adopted an Eulerian approach. Let us introduce $1_{\Omega_{f}}$, the characteristic function of the fluid domain $\Omega_{f}$, as an additional unknown in the interval $] t_{i}, t_{i+1}[$. One can extend each fluid individual problem to the whole computational domain [44] as follows.

Let us note $\chi=\mathcal{V} \times \mathcal{P} \times \mathcal{S} \times \mathcal{L}$ the space containing the whole set of variables of the problem expressed in the fluid. The weak form of this problem can be written as: 


$$
\int_{\Omega_{f}} A(X, Y) d \Omega_{f}=\int_{\Omega_{f}} L(Y) d \Omega_{f}
$$

where $(X, Y) \in \chi^{2}$ and $A$ is the suitable operator. Using the characteristic function $1_{\Omega_{f}}$, problem (38) can be extended to the whole computational domain:

$$
\begin{aligned}
& \int_{\Omega} 1_{\Omega_{f}} A(X, Y)+\left(1-1_{\Omega_{f}}\right) a(X, Y) d \Omega \\
& =\int_{\Omega} 1_{\Omega_{f}} L(Y) d \Omega
\end{aligned}
$$

where $a$ is the generalization to $\chi$ of the velocity-pressure extension method developed in [44], ensuring continuity of the velocity and normal stresses field [45]. Determining the characteristic function for each time step is not detailed here, since it has been the object of a previous work [23]. The characteristic function itself becomes an unknown function which can be approximated using a finite element technique. For instance, one can choose $1_{\Omega_{f}}^{h}=\Pi^{h} 1_{\Omega_{f}}$ where $\Pi^{h}$ is the projection operator onto the piecewise constant space which leads to a V.O.F. method: $1_{\Omega_{f}}^{h}=\operatorname{vol}\left(K \cap \Omega_{f}\right) / \operatorname{vol}(K)$. Finally, moving free surfaces were computed by solving the transport equation:

$$
\frac{d 1_{\Omega_{i}}}{d t}=0
$$

by the space-time formulation.

\subsection{Large number of degrees of freedom and parallel computation}

To capture the different phenomena that we described previously, fine meshes are required, and therefore computations involve a large number of degrees of freedom $4 \times$ NbNodes $+7 \times$ NbElements $\times$ NbModes + NbElements per time slab), and parallel computation is required. The parallel strategy adopted [46] uses the MPI standard programming library and will not be described here. We can briefly say that it is based on a global master-slave program, where SPMD (Single Process, Multiple Data) modules were introduced. Resolution of the non-symmetrical systems from the proposed formulation were done in parallel, using the PETSC (Portable, Extensible Toolkit for Scientific Computation) library, using the GMRES method, with an incomplete LU preconditioning [47].

\section{Numerical results}

In this section, we start by studying standard benchmark start-up flow problems in complex geometrical flows like the start-up shear and contraction-expansion flows, to finally focus on die swell and injection moulding in which compressibility and free surface is studied in both simple geometry and industrial configurations. In all cases, the degree for the time interpolation is set to 0. For each mode $i$ in the viscoelastic spectrum (classified from fast to slow modes with increasing $i$ ), time step Deltat is defined as: 


$$
\begin{array}{r}
\min \left(\frac{1}{\overline{\bar{\gamma}}}, \frac{\theta_{b i}}{10}\right) \text { if } t<10 \theta_{b i} \\
\min \left(\frac{1}{\dot{\bar{\gamma}}}, \frac{\theta_{b i+1}}{10}\right) \text { if } t \geq 10 \theta_{b i}
\end{array}
$$

where $t$ is the elapsed physical time (starting from rest) and $\dot{\bar{\gamma}}$ the shear rate.

Computations were performed in a two-processor machine, except for the injection moulding case, where 4 processors were used.

\subsection{Start-up shear flow}

As a validation example, let us consider orientation under pure shear flow [48] We considered a square domain, $[0,1] \times[0,1]$, with a linear velocity field imposed, $\vec{v}=(0.01+y, 0)$. On the inflow boundary $\left(\partial \Omega_{i n}, x=0\right)$, an isotropic orientation is imposed, $s=I / 2$. To represent the orientation state at each element, we used an ellipse whose semiaxes lengths are the eigenvalues of the associated orientation tensor, and whose directions correspond to its eigenvectors. Relaxation time was considered sufficiently large $(1000 s)$, so that the equation to be solved was similar to the one from Chinesta et al. [48]:

$$
\frac{\partial s}{\partial t}+\vec{v} \cdot \nabla s-\nabla \vec{v} \cdot s-s \cdot \nabla \vec{v}^{T}+2[\epsilon(\vec{v}): s] s=0
$$

In figure 1 one observes that the solution is in agreement with the one obtained by Chinesta et al. [48] with a semi-lagrangian strategy, which is quite good, despite the use of a coarse mesh of approximatively 20 elements in each direction, since particles tend to be oriented in the direction of the flow, as expected in a shear flow. On the right, the results for a similar 3D test case are presented.

\subsection{Contraction/expansion flow}

Let us now consider the start-up flow through a symmetric planar 20:3:20 constriction, with slit height $h=1.5 \mathrm{~mm}$ and slit length $l=1.5 \mathrm{~mm}$. The upstream and downstream regions have lengths of $L=20 \mathrm{~mm}$, and at the inlet a flow rate corresponding to a mean velocity $\bar{V}=25.8 \mathrm{~mm} / \mathrm{s}$ within the slit was imposed, as well as zero normal stresses at the outlet. The flow rate is imposed during $2.8 \mathrm{~s}$ and then we stopped the feeding, letting the material relax back to equilibrium. No-slip boundary conditions were specified at the wall and symmetry at the centerline. Schematic geometry and mesh are shown in figure 2 (with 4609 nodes and 12932 elements). The material (LLDPE) is considered compressible and viscoelastic, obeying the differential multi-mode Pom-Pom model with 5 relaxation modes. The Pom-Pom model parameters are detailed on table 1.

To characterize flow elasticity, one defines the viscosity averaged Weissenberg number:

$$
W e=\bar{\theta}_{b} \dot{\gamma}_{a}
$$

where $\dot{\gamma}_{a}=\bar{V} / h$ is the apparent shear rate and $\bar{\theta}_{b}$ is the averaged orientation relaxation time for the material defined as: 


$$
\bar{\theta}_{b}=\frac{\sum_{i=1}^{m} \theta_{b i}^{2} G_{i}}{\sum_{i=1}^{m} \theta_{b i} G_{i}} .
$$

In complex flows, a Weissenberg number cannot be easily defined. We probably need to consider a local Weissenberg number. From the values given above, the averaged Weissenberg number $W e$ reached 20.8, but it was much higher locally. In what concerns computation data, we performed 705 time steps to reach the steady-state, a computation time of $3 \mathrm{~h} 27 \mathrm{~min} 32 \mathrm{~s}$ with 2 processors.

In figure 3 we display the computed streamlines for different time steps. During the flow, the downstream vortex decreases, whereas the upstream vortex increases in size. The influence of the compressible behavior of the material can also be observed through the pressure plot profile (figure 4). It shows that a certain time is required to reach the steady-state, which depends on the viscoelastic component, but also on the isothermal compressible coefficient. All results are in good agreement with numerical results and experimental observations given in [49] in which the same test case was computed and compared to experiments.

Furthermore, a viscous incompressible versus viscoelastic compressible comparison was performed, using a Carreau law fitting. We observe that the amplitude of the time interval to reach the steady-state is largely underestimated in the viscous incompressible computation.

Stretch (figure 5) and orientation (figure 6) of the different modes are illustrated, where we can see their increase and subsequent relaxation. We remark that at $4 \mathrm{~s}$ some modes have not completely relaxed, whereas others have reached equilibrium.

\subsection{Swell by gravity}

To introduce problems with free surfaces, we present the flow of a 3D jet emerging from a square die. We consider that the fluid falls by gravity into an air-filled cavity of rectangular shape section. The no-slip condition was imposed on the wall of the die. Geometry test data is pictured in figure 7 , as well as its finite element discretization (mesh of 13750 nodes and 69395 elements). Two symmetry plans were taken into account, since our purpose was not to capture eventual instabilities, but to compare the response of two types of materials under this situation. Two different materials were thus considered: a viscoelastic versus a viscous fluid. Material properties considered are displayed in table 2.

The degree of swelling obtained numerically in both cases is shown in figure 8 . We observe that when the fluid is still inside the slit, the difference between the two cases is very small. Nevertheless, at a later time, after exiting the outflow boundary, differences become noticeable. Furthermore, the effect of gravity is present in both situations: it pulls the fluid down, causing a downstream reduction in the jet diameter. In this example, computation time was $4 \mathrm{~h} 57 \mathrm{~min} 03 \mathrm{~s}$ (173 time steps) on 2 processors.

\subsection{Injection moulding flows}

The following case is the filling and post-filling of a biomedical implant (figure 9), with a constant flow rate during the filling stage, a commutation towards a packing pressure for the holding stage and then cooling inside the mold. All process 
parameters and material data are given in table 3.

Three computations were performed in a mesh with 23354 nodes and 126427 elements: one using a viscous incompressible material behavior, one considering a viscous compressible behavior, and finally one taking into account viscoelasticity. Computation times of the whole injection molding cycle were: in the viscous compressible case, 1 day and 19 hours in 8 processors; in the viscoelastic compressible case, 1 day and 16 hours in 8 processors. Each processor is a Pentium IV 2.8Ghz and $1 \mathrm{~Gb}$ RAM, linked to the other processors by a Myrinet network. We underline the fact that, given the number of elements, the viscoelastic computation could not be performed with only one processor.

In this case, we compare simulations performed for both compressible and incompressible behavior consideration. In the incompressible case, we supposed that the density is constant and computed at the atmospheric pressure and injection temperature $\left(\rho=\rho\left(P_{a t m}, T_{i n j}\right)\right)$. Figure 9 shows the evolution of the material in the cavity, as well as the evolution of the flow rate at the inlet. We notice that during the filling stage, no difference is observed in the filling rates evolution.

Figure 10 shows the pressure trace, numerically measured in one point of the part (transducer position at the entrance of the massive part) for both compressible and incompressible cases. We observe that there is a substantial difference, mainly during cooling. In the incompressible case, the abrupt increase at the end of filling, followed the same decrease at the end of packing indicates that there is no transient period for the pressure to attain the packing value. Furthermore, it becomes automatically homogeneous in the part.

Computations were also performed using a viscoelastic compressible model. Influence of material viscoelasticity is not observed generally in the pressure profile, which remains globally the same than in the viscous compressible case. However, during the filling stage, when the pressure is still rather small, the effects of the extra-stress may be observed in the pressure trace. Nevertheless, we can see its influence in the internal stress distribution. Figure 11 illustrates the first principal stress field on the part throughout the process $\left(N_{1}=\sigma_{1}-\sigma_{2}\right)$. When we compare these results with the ones from the literature, we conclude that they follow a good pattern: during filling, molecules located centered molecules are less oriented than the ones closer to the wall, due to the low shear rate, giving rise to lower flow-induces stresses and lower birefringence; at the end of filling, the stresses tend to relax, since velocity reduces drastically; during packing, flow-induced stresses still exist, either frozen or due to materials motion and increase of the relaxation times; at the end of cooling, stresses are frozen-in in the part in regions near the wall. More accurate results could be obtained using a finer mesh, especially near the surface. Furthermore, we believe that quantitative results could be compared to experimental works if a model of the solid state closer to the real material behavior had been considered.

Thus, an orientation distribution is established during the filling stage. Figure 12 shows this distribution for the last mode chosen. Due to its high orientation relaxation time, these orientations did not relax in the processus duration and are present at the ejection moment. A similar analysis can be done for the stretch evolution (figure 13). 


\section{Conclusion}

Computational methods used for computing free surface flows of viscoelastic fluids were described. Methods used involved space-time finite and mixed finite-element methods, as well as parallel computation. The material behavior models were chosen from literature to take into account for material density evolution and extrastress computation, based on polymer engineering works.

The objective of this paper was to show the actual capability of $3 \mathrm{D}$ numerical modelling of transient compressible viscoelastic flows involving moving free surfaces. It has been done by considering sophisticated models: multimode pompom and Tait laws. Moreover, the cost of the computation was accounted in the choice of numerical approximations.

Suitability of the method and correctness of the developments are demonstrated in the results obtained for simple geometries. Simulations performed on complex flows show a good agreement with literature. The underlying application aimed in this work is the injection moulding process. A unique model as the compressible viscoelastic proposed here would lead to an accurate prediction of the compaction stage as well as the residual stresses prediction.

Further work is in progress to consider thermo-viscoelastic-compressibility, as well as more accurate testing on complex geometries to improve our model.

Acknowledgements This study is part of the REM3D project including Atofina, Dow Chemicals, Essilor, FCI, Plastic Omnium, Schneider Electric, Snecma Moteurs, Transvalor and the CEMEF. The first author thanks the financial support of the Portuguese Science and Technology Foundation under the scholarship SFRH/ BD/ 3381/ 2000.

\section{References}

1. A. Poitou and A. Ammar, A molecular model for flow induced crystallization of polymers, Macromolecular Symposia, 185, 243-255 (2002)

2. G.W.M. Peters, F.H.M. Swartjes and H.E.H. Meijer, A Recoverable Strain based Model for Flow-induced Crystallization, Macromolecular Symposia, 185, 277-292 (2002)

3. R. Keunings, Advances in the Computer Modeling of the Flow of Polymeric Liquids, Computational Fluid Dynamics Journal, 9, 449-458 (2001)

4. F.P.T. Baaijens, M.A. Hulsen and P.D. Anderson, The use of mixed finite element methods for viscoelastic fluid flow analysis, Encyclopedia of Computational Mechanic, John Wiley \& Sons, 3, 481-498 (2004)

5. R.G.M. van Os and T. N. Phillips, Efficient and stable spectral element methods for predicting the flow of an XPP fluid past a cylinder, Journal of Non-Newtonian Fluid Mechanics, 129-3, 143-162 (2005)

6. R.J. Poole, M.A. Alves, P.J. Oliveira and F.T. Pinho, Plane sudden expansion flows of viscoelastic liquids, Journal of Non-Newtonian Fluid Mechanics, 146, 79-91 (2007)

7. N. Clemeur, R.P.G. Rutgers and B. Debbaut, Numerical evaluation of three dimensional effects in planar flow birefringence, Journal of Non-Newtonian Fluid Mechanics, 123, 105-120 (2002)

8. I. Sirakov, A. Ainser, M. Haouche and J. Guillet, Three-dimensional numerical simulation of viscoelastic contraction flows using the PomPom differential constitutive model, Journal of Non-Newtonian Fluid Mechanics, 126, 163-173 (2005)

9. R.I. Tanner, A theory of die swell, Journal of Polymer Science, 8, 2067-2078 (1970)

10. M.J. Crochet and R. Keunings, Finite element analysis of die swell of a highly elastic fluid, Journal of Non-Newtonian Fluid Mechanics, 10, 339-356 (1982)

11. R. Keunings, An algorithm for the simulation of transient viscoelastic flows with free surfaces, Journal of Computational Physics, 62, 199-220 (1986) 
12. R. Keunings and D.W. Bousfield, Analysis of surface tension driven levelling in horizontal viscoelastic films, Journal of Non-Newtonian Fluid Mechanics, 22, 219-233 (1987)

13. J. Cormenzana, A. Ledda, M. Laso and B. Debbaut,Calculation of free surface flows using CONNFESSIT, Journal of Rheology, 45, 237-258 (2001)

14. K.S. Sujatha, H. Matallah, M.J. Banaai and M.F. Webster, Computational predictions for viscoelastic filament stretching flows: ALE methods and free-surface techniques (CM and VOF), Journal of Non-Newtonian Fluid Mechanics, 137, 81-102 (2006)

15. J. Etienne, E.J. Hinch and J. Li, A Lagrangian-Eulerian approach for the numerical simulation of free-surface flow of a viscoelastic material, Journal of Non-Newtonian Fluid Mechanics, 136, 157-166 (2006)

16. M.F. Tomé, N. Mangiavacchi, J.A. Cuminato, A. Castelo and S. McKee, A finite difference technique for simulating unsteady viscoelastic free surface flows, Journal of Non-Newtonian Fluid Mechanics, 106, 61-106 (2002)

17. G.S. de Paulo, M.F. Tomé and S. McKee, A marker-and-cell approach to viscoelastic free surface flows using the PTT model, Journal of Non-Newtonian Fluid Mechanics, 147, 149-174 (2007)

18. R. Guénette and M. Fortin, A new finite element method for computing viscoelastic flows, Journal of Non-Newtonian Fluid Mechanics, 60, 27-52 (1995)

19. T.C.B. McLeish and R.G. Larson, Molecular constitutive equations for a class of branched polymers: the pom-pom polymer, Journal of Rheology, 42, 81-110 (1998)

20. K. Lee, M.R. Mackley, T.C.B. Mcleish, T.M. Nicholson and O. Harlen, Experimental observation and numerical simulation of transient stress fangs within flowing molten polyethylene, Journal of Rheology, 45, 1261-1277 (2001)

21. T. Coupez, On the utility of the mesh in computational material forming, 6 th International ESAFORM Conference on Material Forming, V. Brucato, Nuova Ipsa Editore, 599-602 (2003)

22. J.M. Floryan and H. Rassmussen, Numerical Methods for Viscous Flows with Moving Boundaries, Applied Mechanics Review, 42, 323-341 (1989)

23. S. Batkam, J. Bruchon and T. Coupez, A space-time discontinuous Galerkin method for convection and diffusion in injection moulding, International Journal of Forming Processes, 7, 11-33 (2004)

24. F.H. Harlow and A.A. Amsden, A Numerical Fluid Dynamics Calculation Method for All Flow Speeds, Journal of Computational Physics, 8, 197-213 (1971)

25. R.I. Issa, A.D. Gosman and A.P. Watkins, The Computation of Compressible and Incompressible Free Recirculating Flows by a Non-Iterative Implicit Scheme, Journal of Computational Physics, 62, 66-82 (1986)

26. T. Yabe and P.Y. Wang, Unified Numerical Procedure for Compressible and Incompressible Flows, Journal of the Physical Society of Japan, 60,2105-2108 (1991)

27. O.C. Zienkiewicz and J. Wu, A General Explicit or Semi-Explicit Algorithm for Compressible and Incompressible Flows, International Journal for Numerical Methods in Engineering, 35, 475-479 (1992)

28. G. Hauke and T.J.R. Hughes, A Unified Approach to Compressible and Incompressible Flows, Computer Methods in Applied Mechanics and Engineering, 113, 389-395 (1994)

29. N. Nonaka and T. Nakayama, A Unified Method for the Numerical Analysis of Compressible and Incompressible Viscous Flows, Computational Mechanics, 19, 369-376 (1996)

30. N. Nigro, M Storti and S. Idelsohn, A General Algorithm for Compressible and Incompressible Flow. Stability Analysis and Explicit Time Integration, International Journal of Numerical Methods for Heat and Fluid Flow, 7, 141-168 (1997)

31. F. Belblidia, I.J. Keshtiban and M.F. Webster, Stabilised computations for viscoelastic flows under compressible implementations, Journal of Non-Newtonian Fluid Mechanics, 134, 56-76 (2006)

32. I.J. Keshtiban, F. Belblidia and M.F. Webster, Numerical simulation of compressible viscoelastic liquids, Journal of Non-Newtonian Fluid Mechanics, 122, 131-146 (2004)

33. I.J. Keshtiban, F. Belblidia and M.F. Webster, Computation of incompressible and weakly-compressible viscoelastic liquids flow: finite element/volume schemes, Journal of NonNewtonian Fluid Mechanics, 126, 123-143 (2005)

34. R. Valette, M.R. Mackley and G. Hernandez Fernandez del Castillo, Matching time dependent pressure driven flows with a Rolie Poly numerical simulation, Journal of Non-Newtonian Fluid Mechanics, 136, 118-125 (2006)

35. L. Silva, J.-F. Agassant and T. Coupez, Three dimension injection molding simulation, Injection molding: fundamentals and applications, M. Kamal and A. Isayev and S.-H. Liu eds, Hanser-Verlag (2008) 
36. C. A. Hieber, Modelling the PVT Behavior of Isotactic Polypropylene, International Polymer Processing, 12, 249-256 (1997)

37. W.M.H. Verbeeten, G.W.M. Peters and F.P.T. Baaijens, Viscoelastic analysis of complex polymer melt flows using the eXtended PomPom model, Journal of Non-Newtonian Fluid Mechanics, 108, 301-326 (2002)

38. M. Fortin and A. Fortin, A new class for the FEM simulation of viscoelastic flows, Journal of Non-Newtonian Fluid Mechanics, 32, 341-357 (1989)

39. O. M. Coronado, D. Arora, M. Behr, M. Pasquali, Four-field Galerkin/least-squares formulation for viscoelastic fluids, Journal of Non-Newtonian Fluid Mechanics, 140, 132-144 (2006)

40. D.N. Arnold, F. Brezzi and M. Fortin, A stable finite element for Stokes equations, Calcolo, $21,337-344$ (1984)

41. R. Kellog and B. Liu, A finite element method for the compressible Navier-Stokes equations, SIAM Journal of Numerical Analysis, 33, 780-788 (1996)

42. M. Fortin, H. Manouzi and A. Soulaimani, On finite element approximation and stabilization methods for compressible viscous flows, International Journal for Numerical Methods in Fluids, 17, 477-499 (1993)

43. T. Coupez and S. Marie, From a direct solver to a parallel iterative solver in 3-D forming simulation, International Journal Of Supercomputer Applications And High Performance Computing, 11, 277-285 (1997)

44. E. Pichelin and T. Coupez, Finite element solution of the $3 \mathrm{D}$ mold filling problem for viscous incompressible fluid, Computer Methods in Applied Mechanics and Engineering, 163, 359-371 (1998)

45. J. Bruchon and T. Coupez, A numerical strategy for the direct 3D simulation of the expansion of bubbles into a molten polymer during a foaming process, International Journal for Numerical Methods in Fluids, 57, 977-1003 (2008)

46. H. Digonnet and T. Coupez, Object-Oriented Programming for "fast-and-easy" development of parallel applications in forming processes simulation, Second MIT Conference on Computational Fluid and Solid Mechanics, K.J. Bathe ed, Elsevier, 1922-1924 (2003)

47. S. Balay, K. Buschelman, V. Eijkhout, W.D. Gropp, D. Kaushik, M.G. Knepley, L. Curfman McInnes; B.F. Smith and H. Zhang, PETSc Users Manual, ANL-95/11 - Revision 2.1.5, Argonne National Laboratory (2004)

48. F. Chinesta, A. Poitou and R. Torres, A semi-lagrangian strategy to predict the fiber orientation in the steady flows of reinforced thermoplastics, Computer Methods in Applied Mechanics and Engineering, 189, 233-247 (2000)

49. R. Valette, M.R. Mackley and G. Hernandez Fernandez del Castillo, The effect of compressibility, shear thinning, viscoelasticity and absolute pressure on time dependent pressure driven polymer melt flow, Proc. XIVth Int. Conf. Rheol. (2004) 

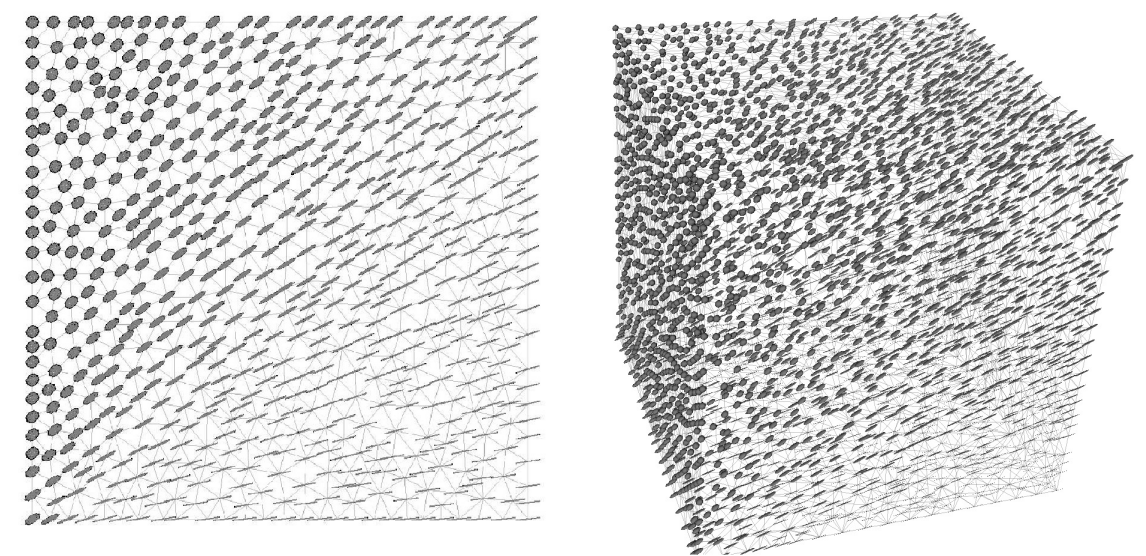

Fig. 1 Orientation solution in 2D and 3D, for a pure shear flow.

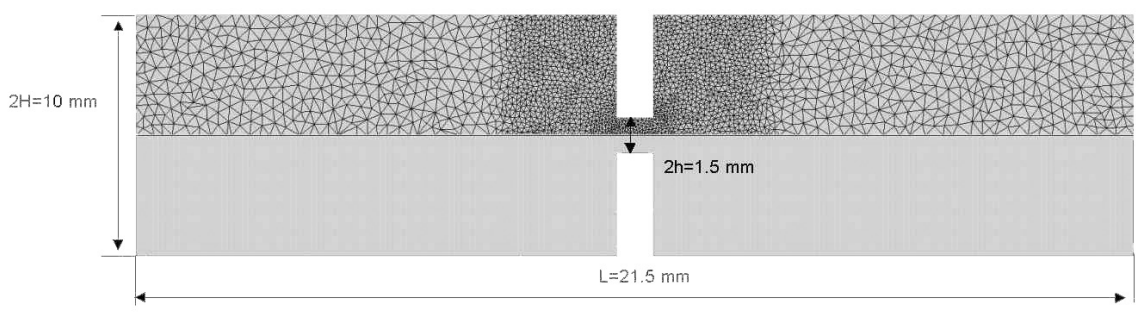

Fig. 2 Geometry and mesh of the contraction/expansion test. 


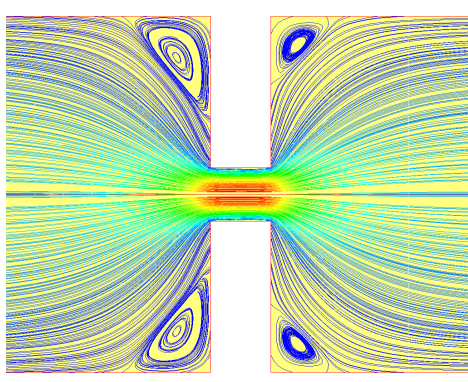

(a)

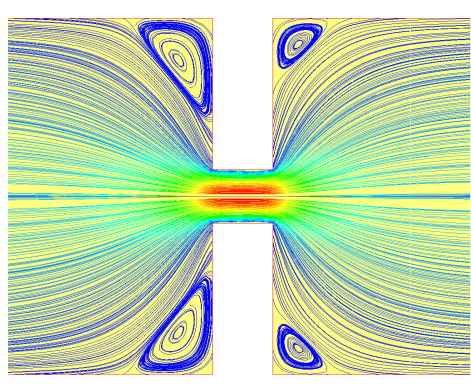

(c)

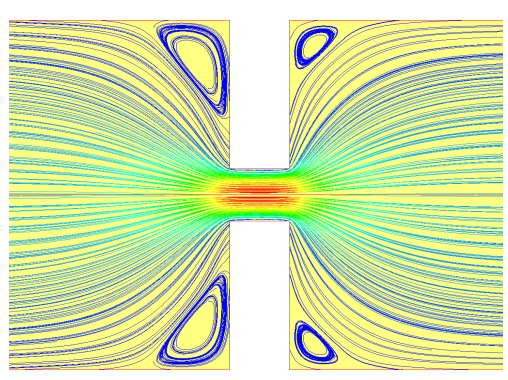

(b)

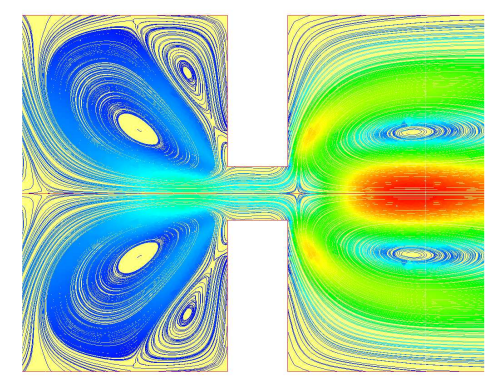

(d)

Fig. 3 Streamlines for the contraction/expansion test, at different time steps ( $\mathrm{a}) t=0, \mathrm{~b})$ $t=\theta_{b}$, c) $t=10 \theta_{b}$, d) steady-state).

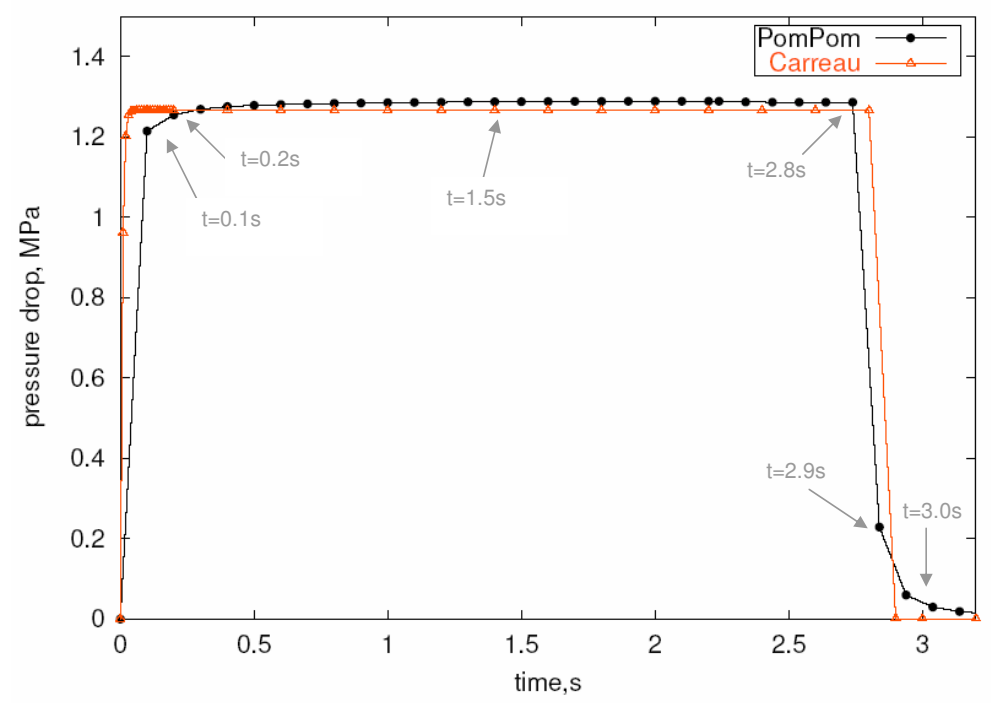

Fig. 4 Pressure drop throughout time for the contraction/expansion test. 


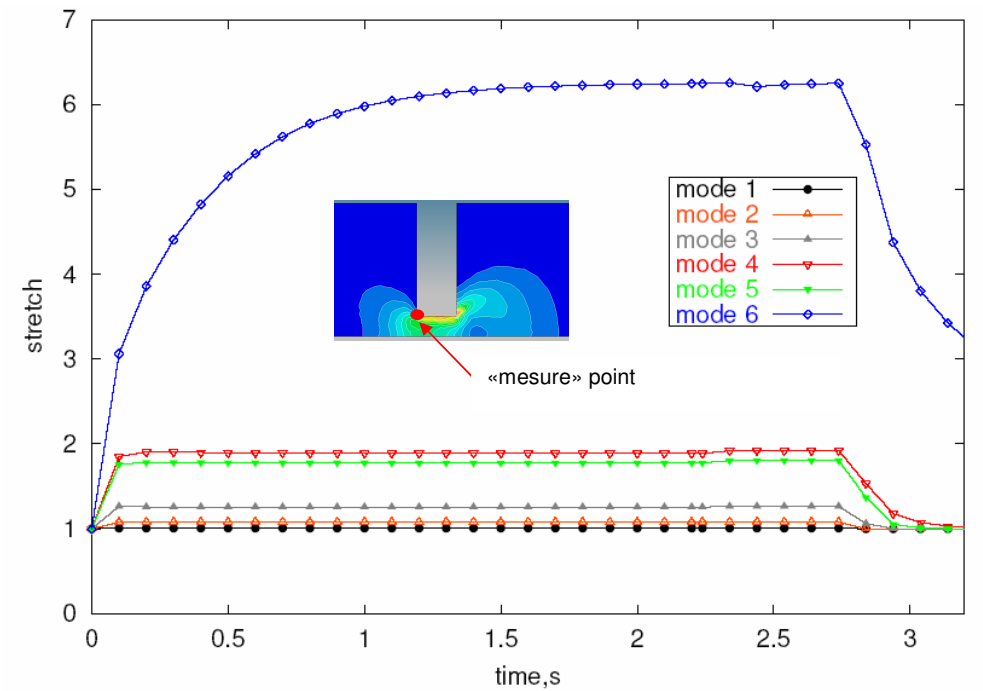

Fig. 5 Stretch evolution in the contraction-expansion device, for the different modes. 
$\mathrm{t}=0.1 \mathrm{~s}$
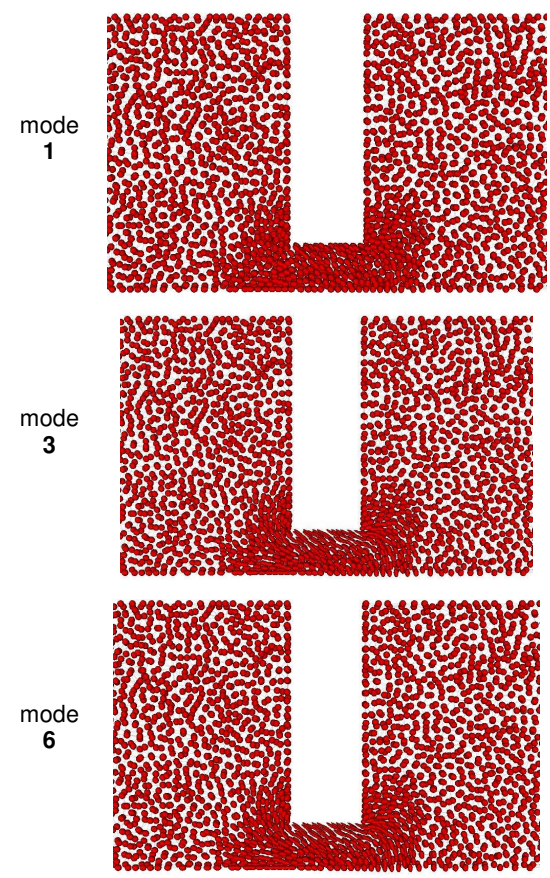

$t=1 \mathrm{~s}$

mode 1

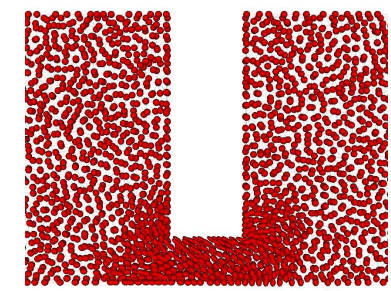

mode 3

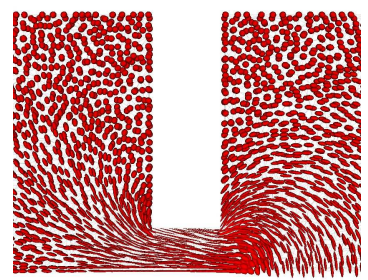

mode 6

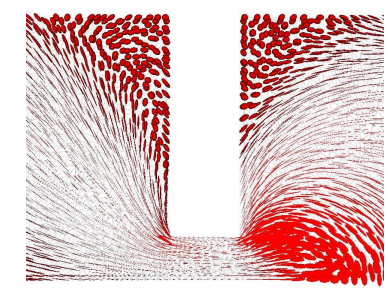

$t=0.1 \mathrm{~s}$
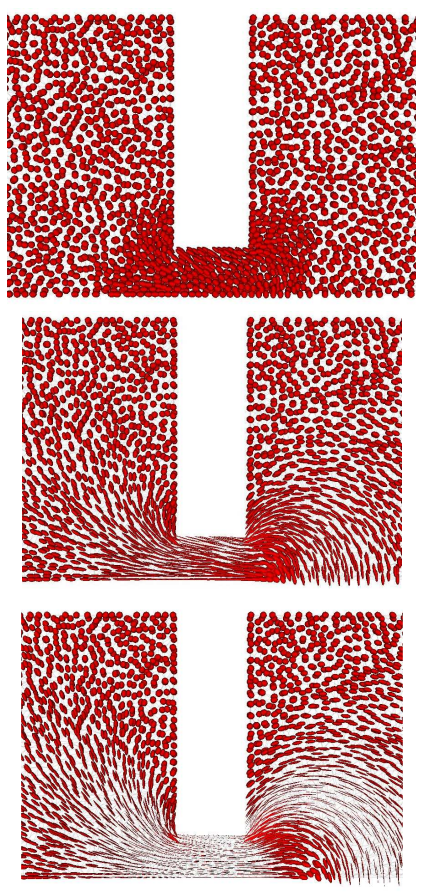

$t=3 s$
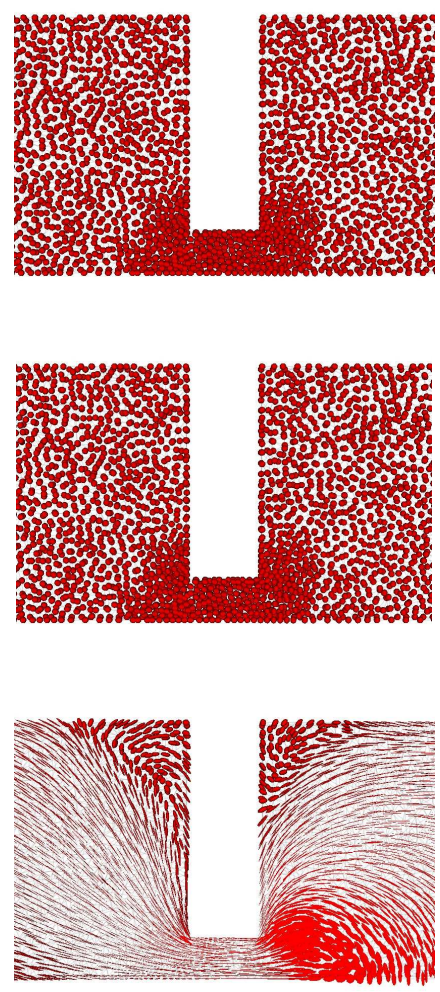

Fig. 6 Orientation profile evolution in the contraction-expansion device, for three different modes. 


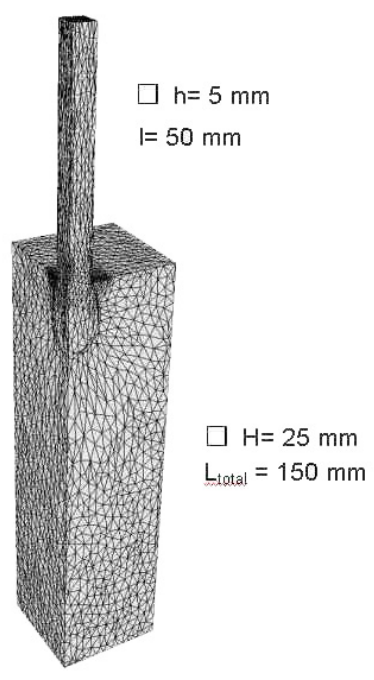

Fig. 7 Geometry of the swell under gravity test. 


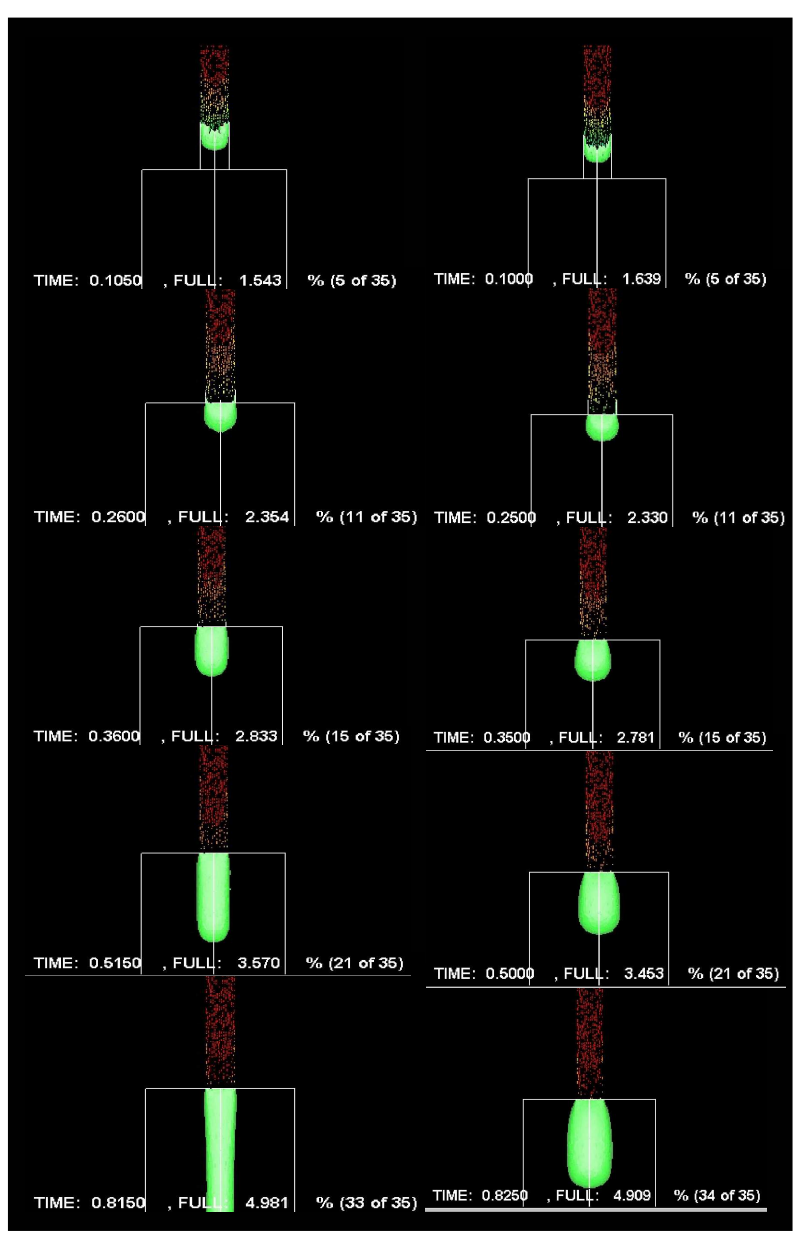

Fig. 8 Evolution of the flow front throughout time for the swell test. On the left, the viscoelastic solutions, on the right the newtonian ones. 


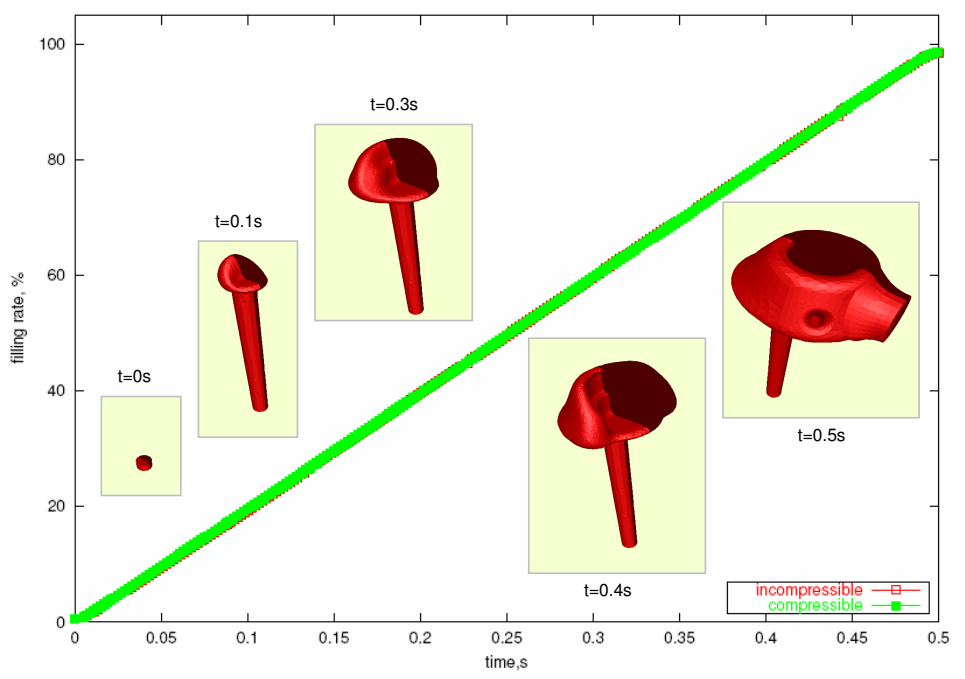

Fig. 9 Filling and flow rate function of time for the biomedical implant injection moulding test case.

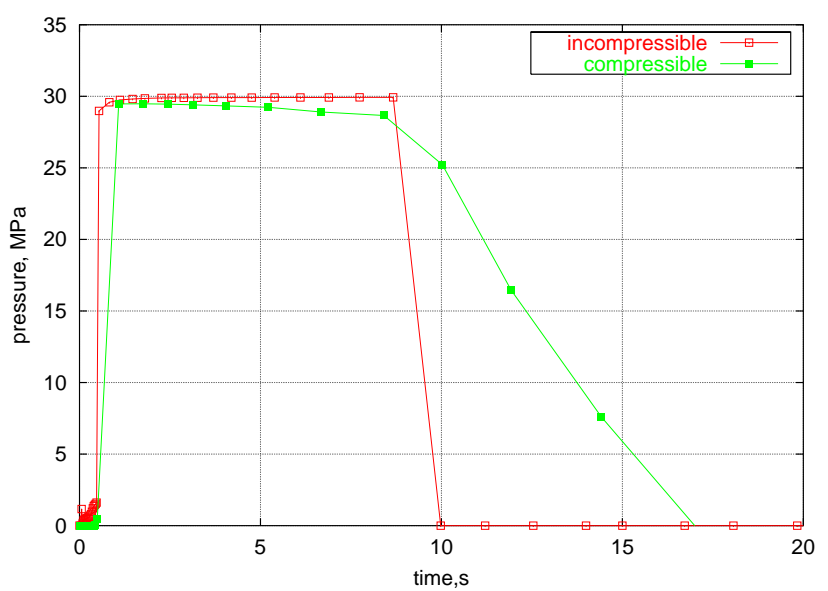

Fig. 10 Evolution of pressure in the cavity as function of time, for the compressible and incompressible test cases. 


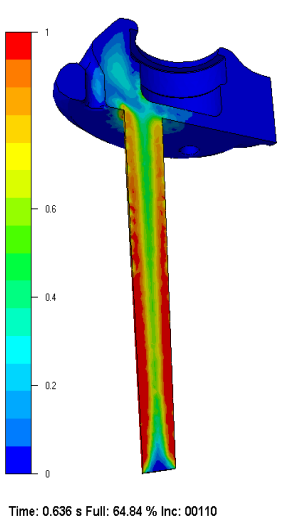

(a) $t=0.6 s$, filling

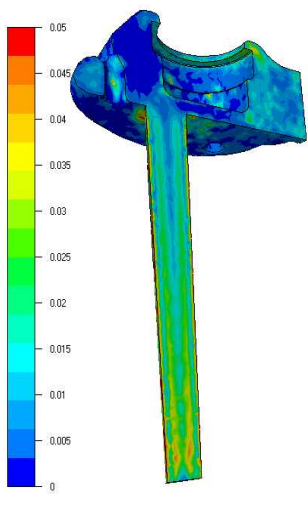

(c) $\mathrm{t}=2.8 \mathrm{~s}$, packing

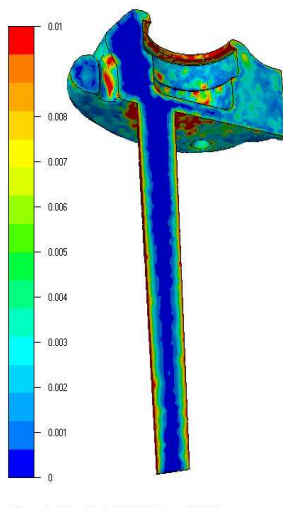

(e) $t=8.8 \mathrm{~s}$, cooling

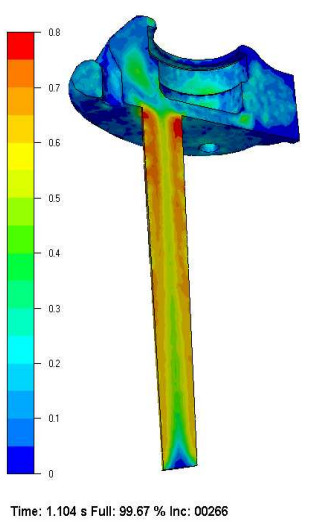

(b) $\mathrm{t}=1 \mathrm{~s}$, end filling

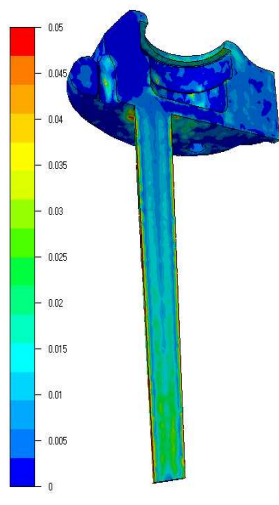

(d) $\mathrm{t}=4.8 \mathrm{~s}$, cooling

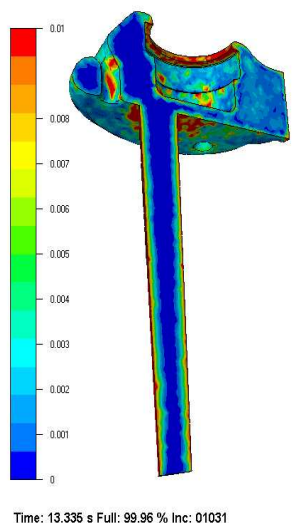

(f) $t=20$ s end cooling

Fig. 11 First principal stress difference distribution in the part, $N_{1}=\sigma_{1}-\sigma_{2}$. 


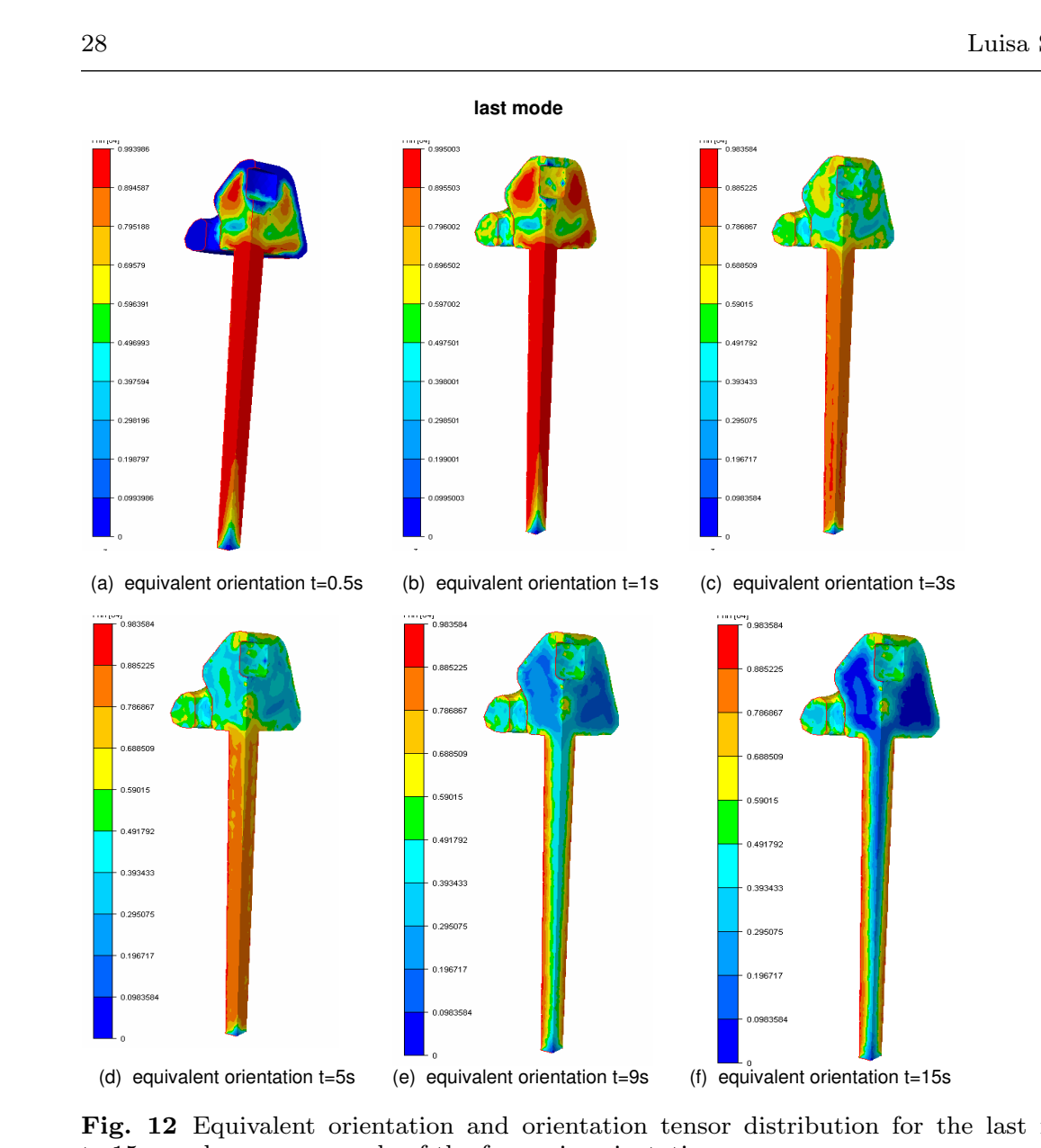


Title Suppressed Due to Excessive Length

29

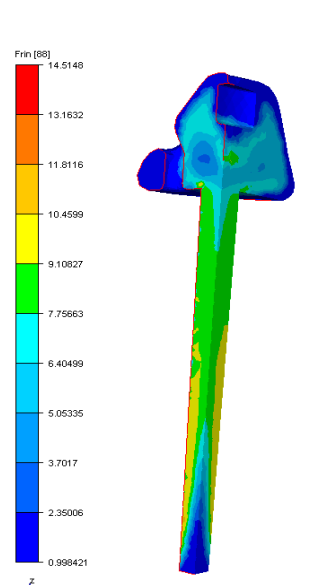

(a) equivalent stretch $\mathrm{t}=0.5 \mathrm{~s}$

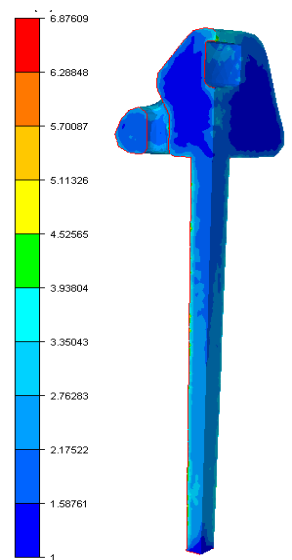

(d) equivalent stretch $\mathrm{t}=5 \mathrm{~s}$

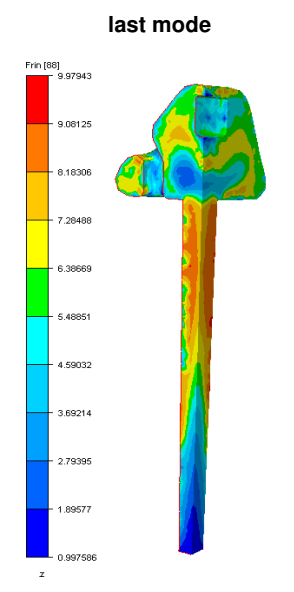

(b) equivalent stretch $\mathrm{t}=1 \mathrm{~s}$

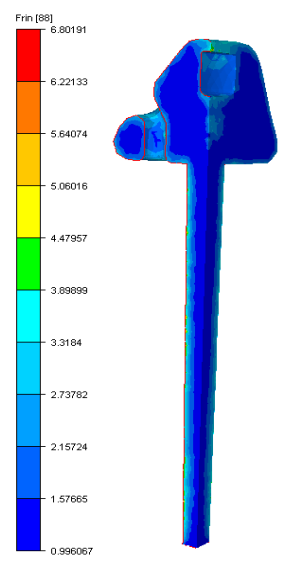

(e) equivalent stretch $\mathrm{t}=9 \mathrm{~s}$

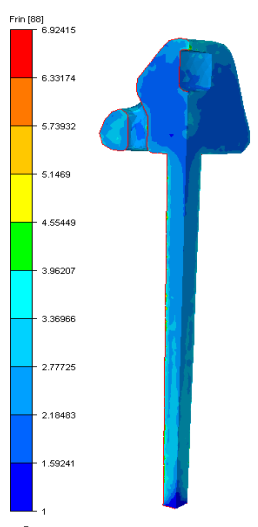

(c) equivalent stretch $\mathrm{t}=3 \mathrm{~s}$

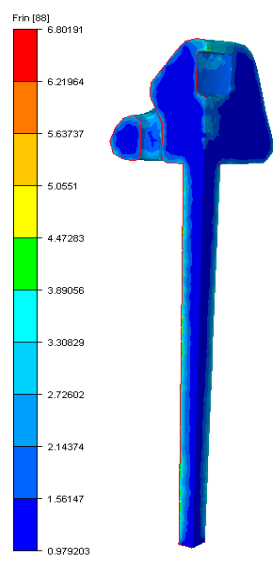

(f) equivalent stretch $t=15 \mathrm{~s}$

Fig. 13 Stretch distribution for the last mode; values of the frozen-in stretch reach locally 6.8 . 


\begin{tabular}{|c|c|c|c|c|}
\hline$N$ & $\theta_{b, i}(\mathrm{~s})$ & $G_{i}(\mathrm{~Pa})$ & $q_{i}$ & $\theta_{b, i} / \theta_{s, i}$ \\
\hline 1 & $1.14 \mathrm{E}-02$ & $9.89 \mathrm{E}+06$ & 1 & 2 \\
2 & $5.16 \mathrm{E}-02$ & $3.77 \mathrm{E}+06$ & 1 & 2 \\
3 & $2.35 \mathrm{E}-01$ & $7.48 \mathrm{E}+05$ & 2 & 2 \\
4 & $1.07 \mathrm{E}+00$ & $1.40 \mathrm{E}+05$ & 4 & 2 \\
5 & $4.84 \mathrm{E}+00$ & $1.52 \mathrm{E}+04$ & 5 & 2 \\
\hline$\chi_{p}$ (constant) & \multicolumn{5}{|c|}{$1.50 \mathrm{E}-11 \mathrm{~Pa}^{-1}$} \\
\hline
\end{tabular}

Table 1 Constitutive parameters of LLDPE at $190{ }^{\circ} \mathrm{C}$.

\begin{tabular}{|ll|rl|}
\hline \multicolumn{1}{|l|}{ Viscous fluid } & & Viscoelastic fluid \\
\hline$\rho$ & $=1.0 \mathrm{E}+06 \mathrm{~g} . \mathrm{m}^{-3}$ & $\rho$ & $=1.0 \mathrm{E}+06 \mathrm{~g} . \mathrm{m}^{-3}$ \\
$\eta$ & $=1.0 \mathrm{E}+01 \mathrm{~Pa} . \mathrm{s}$ & $\eta$ & $=1.0 \mathrm{E}+01 \mathrm{~Pa} . \mathrm{s}$ \\
& & $G$ & $=1.0 \mathrm{E}+03 \mathrm{~Pa}$ \\
& & $\theta_{b}$ & $=5.0 \mathrm{E}-03 \mathrm{~s}$ \\
& & $\theta_{s}$ & $=2.5 \mathrm{E}-03 \mathrm{~s}$ \\
& $q$ & $=5$ \\
\hline
\end{tabular}

Table 2 Material parameters used to the swell test.

\begin{tabular}{|c|c|}
\hline \multicolumn{2}{|r|}{ Process parameters } \\
\hline Mould temperature & $=50^{\circ} \mathrm{C}$ \\
\hline Material temperature & $=220^{\circ} \mathrm{C}$ \\
\hline Filling flow rate & $=7.0 \mathrm{E}-06 \mathrm{~m}^{3} \cdot \mathrm{s}^{-1}$ \\
\hline Packing pressure & $=3.0 \mathrm{E}+07 \mathrm{~Pa}$ \\
\hline Holding time & $=9 \mathrm{~s}$ \\
\hline Cooling time & $=11 \mathrm{~s}$ \\
\hline \multicolumn{2}{|r|}{ Material properties } \\
\hline \multicolumn{2}{|l|}{ Tait law: } \\
\hline$b_{1_{l}}$ & $=1.0064 \mathrm{E}-06 \mathrm{~m}^{3} \cdot \mathrm{g}^{-1}$ \\
\hline$b_{2_{l}}$ & $=6.2748 \mathrm{E}-10 \mathrm{~m}^{3} \cdot \mathrm{g}^{-1} \cdot{ }^{o} \mathrm{C}^{-1}$ \\
\hline$b_{3 l}$ & $=1.3957 \mathrm{E}+08 \mathrm{~Pa}$ \\
\hline$b_{4}$ & $=4.0564 \mathrm{E}-03^{\circ} \mathrm{C}^{-1}$ \\
\hline$b_{1 s}$ & $=1.0049 \mathrm{E}-06 \mathrm{~m}^{3} \cdot \mathrm{g}^{-1}$ \\
\hline$b_{2 s}$ & $=2.3766 \mathrm{E}-10 \mathrm{~m}^{3} \cdot \mathrm{g}^{-1} \cdot{ }^{o} \mathrm{C}^{-1}$ \\
\hline$b_{3 s}$ & $=1.9856 \mathrm{E}+08 \mathrm{~Pa}$ \\
\hline$b_{4_{s}}$ & $=2.1512 \mathrm{E}-03^{\circ} \mathrm{C}^{-1}$ \\
\hline$b_{5}$ & $=9.092 \mathrm{E}+01{ }^{\circ} \mathrm{C}$ \\
\hline$b_{6}$ & $=3.0068 \mathrm{E}-07^{\circ} \mathrm{C} \cdot \mathrm{Pa}^{-1}$ \\
\hline$b_{7}$ & $=0 \mathrm{~m}^{3} \cdot \mathrm{g}^{-1}$ \\
\hline$b_{8}$ & $=0^{\circ} \mathrm{C}^{-1}$ \\
\hline$b_{9}$ & $=0 \mathrm{~Pa}^{-1}$ \\
\hline \multicolumn{2}{|c|}{ Pom-Pom model (6 modes): } \\
\hline$\eta$ & $=1.23 \mathrm{E}+03$ Pa.s \\
\hline$G$ & $=3.3 \mathrm{E}+059.84 \mathrm{E}+043.77 \mathrm{E}+04 \quad 7.48 \mathrm{E}+03 \quad 1.40 \mathrm{E}+031.52 \mathrm{E}+02 \mathrm{~Pa}$ \\
\hline$\theta_{b}$ & $=2.50 \mathrm{E}-031.14 \mathrm{E}-02 \quad 5.16 \mathrm{E}-022.35 \mathrm{E}-01 \quad 1.07 \mathrm{E}-014.84 \mathrm{~s}$ \\
\hline$\theta_{s}$ & $=1.25 \mathrm{E}-035.70 \mathrm{E}-032.58 \mathrm{E}-021.18 \mathrm{E}-015.35 \mathrm{E}-022.42 \mathrm{~s}$ \\
\hline$q$ & \\
\hline
\end{tabular}

Table 3 Process and material parameters used in the injection moulding of the biomedical device, for density and extra-stress evolution. 